\title{
Coarse Moduli Space for Polarized Compact Kähler Manifolds
}

\author{
Dedicated to Professor S. Nakano on his 60th birthday
}

By

Akira FuJIKI*

\begin{abstract}
Albstract
Using the results of our previous papers [5] [8] [9] we shall construct the coarse moduli space for non-uniruled polarized compact Kähler manifolds as a separated complex space.
\end{abstract}

\section{$\S 1$. Statement of $\mathbb{R}$ esull}

(1.1) A polarized compact Kähler manifold is by definition a pair $(X, \omega)$ consisting of a connected compact complex manifold $X$ and a Kähler class $\omega \in$ $H^{2}(X, \mathbb{R})$ on $X$. Here a Kähler class is a class represented by a Kähler form, i.e., the fundamental 2-form associated with a Kähler metric on $X$. An isomorphism of two polarized compact Kähler manifolds $(X, \omega)$ and $\left(X^{\prime}, \omega^{\prime}\right)$ is by definition an analytic isomorphism $\psi: X \rightarrow X^{\prime}$ with $\psi^{*} \omega^{\prime}=\omega$.

Definition. i) A polarized family of compact Kähler manifolds (parametrized by a complex space $S)$ is a pair $(f, \widetilde{\omega})$ consisting of a proper smooth morphism $f: \mathscr{X} \rightarrow S$ of complex spaces with connected fibers and an element $\widetilde{\omega} \in \Gamma\left(S, R^{2} f_{*} \mathbb{R}\right)$ such that

(1) $\widetilde{\omega}$ induces on each fiber $X_{s}:=f^{-1}(s)$ a $\mathbb{K a ̈ h l e r ~ c l a s s ~} \widetilde{\omega}_{s} \in H^{2}\left(X_{s}, \mathbb{R}\right)$, and

(2) $\eta(\widetilde{\omega})=0$, where $\eta=\eta_{f}: \Gamma\left(S, R^{2} f_{*} \mathbb{R}\right) \rightarrow \Gamma\left(S, R^{2} f_{*} \mathcal{O}_{\mathfrak{X}}\right)$ is the homomorphism induced by the natural inclusion $\mathbb{R} \rightarrow \mathcal{O}_{\mathscr{X}}$ of sheaves on $\mathscr{X}$. ((2) is a consequence of (1) when $S$ is reduced (cf. (2.3) and Lemma 1 below).) When no confusion may arise we often call $(f, \widetilde{\omega})$ simply a polarized family.

ii) An isomorphism of two polarized families $(f: \mathscr{X} \rightarrow S, \widetilde{\omega})$ and $\left(f^{\prime}: \mathscr{X}^{\prime}\right.$

Communicated by S. Nakano, September 26, 1983. Revised February 22, 1984.

* Yoshida College, Kyoto University, Kyoto 606, Japan 
$\left.\rightarrow S, \widetilde{\omega}^{\prime}\right)$ is an $S$-isomorphism $\psi: \mathfrak{X} \rightarrow \mathfrak{X}^{\prime}$ of complex spaces such that $\psi^{*} \widetilde{\omega}^{\prime}$ $=\widetilde{\omega}$ in $\Gamma\left(S, R^{2} f_{*} \mathbb{R}\right)$.

(1.2) We denote by $\mathfrak{M}$ the set of isomorphism classes of polarized compact Kähler manifolds. For the simplicity of notation, however, we usually write $(X, \omega) \in \mathfrak{M}$, identifying a polarized compact Kähler manifold $(X, \omega)$ with its isomorphism class.

Let $(f: \mathscr{X} \rightarrow S, \widetilde{\omega})$ be a polarized family of compact Kähler manifolds. Then for each $s \in S\left(X_{s}, \widetilde{\omega}_{s}\right)$ is a polarized compact Kähler manifold. Thus associating with each $s \in S$ the isomorphism class of $\left(X_{s}, \widetilde{\omega}_{s}\right)$ we obtain a natural map $\rho(f, \widetilde{\omega}):|S| \rightarrow \mathfrak{M}$ where $|S|$ denotes the underlying topological space of $S$. We call $\rho(f, \widetilde{\omega})$ the canonical map associated with $(f, \widetilde{\omega})$.

The canonical topology of $\mathfrak{M}$ is by definition the finest topology for which $\rho(f, \widetilde{\omega})$ is continuous for any polarized family $(f, \widetilde{\omega})$ as above; thus a subset $U \cong \mathfrak{M}$ is open if and only if $\rho(f, \widetilde{\omega})^{-1}(U)$ is open in $|S|$ for any $(f, \widetilde{\omega})$.

(1.3) Let $(f: \mathscr{X} \rightarrow S, \widetilde{\omega})$ be a polarized family of compact Kähler manifolds. Let $\nu: T \rightarrow S$ be any morphism of complex spaces. We shall then define the pull-back $\left(f_{T}, \widetilde{\omega}_{T}\right)$ of $(f, \widetilde{\omega})$ by $\nu$. Let $f_{T}: \mathscr{X} \times{ }_{S} T \rightarrow T$ be the induced morphism. Set $\widetilde{\omega}_{T}=\nu^{*} \widetilde{\omega}$, where $\nu^{*} \widetilde{\omega}$ is considered as an element of $\Gamma\left(T, R^{2} f_{T^{*}} R\right)$ via the natural isomorphism $\nu^{*} R^{2} f_{*} \mathbb{R} \cong R^{2} f_{T^{*} \mathbb{R}}$. Then the pair $\left(f_{T}, \widetilde{\omega}_{T}\right)$ is again a polarized family of compact Kähler manifolds; indeed, (1) is clear and (2) follows from the naturality of $\eta_{f}$ (cf. (2.3) below). We call $\left(f_{T}, \widetilde{\omega}_{T}\right)$ the pullback of $(f, \widetilde{\omega})$ by $\nu$.

Let $\mathfrak{\mho} \cong \mathfrak{M}$ be a subset. Then a polarized family $(f, \widetilde{\omega})$ as above is said to be a (polarized) $\widetilde{\mho}$-family if $\rho(f, \widetilde{\omega})(|S|) \subseteq \widetilde{\mho}$. It is clear that if $(f, \widetilde{\omega})$ is

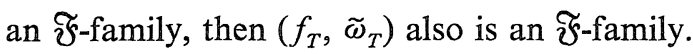

Let An be the category of complex spaces. Then with the definition of the pull-back as above, for any subset $\mathfrak{F} \cong \mathfrak{M}$ we define the contravariant functor $\mathscr{F}:$ An $\rightarrow$ Sets of An into the category of sets by

$\mathscr{F}(S)=$ the set of isomorphism classes of polarized $\mathfrak{\mho}$-families $(f: \mathfrak{X} \rightarrow S, \widetilde{\omega})$ parametrized by $S$.

Definition. Let $\mathfrak{F} \subseteq \mathfrak{M}$ be a subset. Then the coarse $\mathfrak{\Im}-$ moduli space is a pair $(F, \psi)$ consisting of a complex space $F$ and a morphism of functors $\psi$ : $\mathscr{I} \rightarrow h_{F}:=\operatorname{Hom}(, F)$ of $\mathscr{I}$ into the representable functor $h_{F}$ such that 1$)$ the underlying topological space of $F$ is canonically homeomorphic to $\mathfrak{F}$ where the topology of $\mathfrak{F}$ is induced from the canonical topology of $\mathfrak{M}$, and 2) for any complex space $F_{1}$ and a morphism of functors $\psi_{1}: \mathscr{F} \rightarrow h_{F_{1}}:=\operatorname{Hom}\left(, F_{1}\right)$ 
there exists a unique morphism $\tau: F \rightarrow F_{1}$ of complex spaces such that $\psi_{1}=h_{\tau} \psi$ 。

Note that the canonicity in 1 ) means that the natural map $\mathfrak{F} \rightarrow F$ defined by $\psi$ is homeomorphic.

(1.4) Let $X$ be a compact complex manifold. $X$ is said to be uniruled if there exist a compact complex manifold $Y$, a holomorphic vector bundle $E$ of rank $\geqq 2$ on $Y$ and a generically surjective meromorphic map $\pi: \mathbb{P}(E) \rightarrow X$ which is not factored by the projection $\mathbb{P}(E) \rightarrow Y$, where $\mathbb{P}(E)$ is the projective bundle associated to $E$ (cf. [8]). Then our main result is stated as follows.

Theorem. Let $\mathfrak{U}=\{(X, \omega) \in \mathfrak{M} ; X$ is not uniruled $\}$. Then the coarse $\mathfrak{Q}$-moduli space $(A, \psi)$ exists.

Remark 1. Actually, in the course of the proof we specify a subset $\mathfrak{B} \subseteq \mathfrak{M}$ containing $\mathfrak{A}$ such that the coarse $\mathfrak{B}$-moduli space $\left(B, \psi_{B}\right)$ exists except that $B$ is not separated (cf. Theorem 2).

Our proof of theorem follows in main line the method of NarasimhanSimha [24] in which they have shown the existence of the coarse $\Omega$-moduli space (in the reduced category), where $\Re=\left\{(X, \omega) \in \mathfrak{M}: \omega=-c_{1}^{R}(X)\right\}, c_{1}^{R}(X)$ being the real first chern class of $X$.

The arrangement of this paper is as follows. Section 2 is preliminary. In Section 3 we give a kählerian analogue of the separation criterion of Matsusaka-Mumford [22] essentially proved in [9]. Let $\mathfrak{I}=\left\{(X, \omega) \in \mathfrak{M}\right.$; $\mathrm{Aut}_{0} X$ is a complex torus\}. Then in Section 4 we show the cohomological flatness of the relative tangent sheaf for any polarized $\mathfrak{I}$-family (Theorem 1). We construct the local modular family for any $(X, \omega)$ in $\mathfrak{I}$ and study the local structure of $\mathfrak{I}$ in Section 5. Then in Section 6 we define the subspace $\mathfrak{B} \subseteq \mathbb{M}$ and prove Theorem 2 mentioned above using the results of Section 5. Together with the main result of [8] the proof of Theorem is immediate from Theorem 2 (Section 7). Finally in Section 8, as concrete examples we shall give an explicit description of the moduli spaces for complex tori and $\mathbb{K} 3$ surfaces by summarizing the known results in these cases.

The results of this paper were announced in [7] in a somewhat weaker form. In the subsequent paper [12] we shall construct the coarse moduli space for non-uniruled polarized algebraic manifolds as a separated algebraic space.

Our thanks is due to the referee for a simplification of the proof of Proposition 5 . 


\section{§2. Preliminaries}

(2.1) a) Let $S$ be a complex space. Let $G \rightarrow S$ be a morphism of complex spaces. Then $G$ is called a complex Lie group over $S$ if there exist $S$-morphisms $G \times{ }_{S} G \rightarrow G, G \rightarrow G, S \rightarrow G$ defining relative multiplication, inversion, and the identity section respectively satisfying the usual axioms (cf. [23, Def. 0.1] or $[10, \S 1])$. Let $f: \mathscr{X} \rightarrow S$ be a morphism of complex spaces. Then a relative action of $G$ on $\mathscr{X}$ over $S$ is an $S$-morphism $\sigma: G \times{ }_{S} \mathscr{X} \rightarrow \mathscr{X}$ satisfying the usual axiom of actions (cf. [23, Def. 0.3]).

b) Let $f: \mathfrak{X} \rightarrow S$ and $f^{\prime}: \mathscr{X}^{\prime} \rightarrow S$ be proper smooth morphisms of complex spaces. Let $D \mathscr{X} \times{ }_{S} X^{\prime} / S \rightarrow S$ be the relative Douady space associated with $f \times{ }_{S} f^{\prime}$ : $\mathscr{X} \times{ }_{s} \mathfrak{X}^{\prime} \rightarrow S$. Let $\operatorname{Isom}_{s}\left(\mathscr{X}, \mathfrak{X}^{\prime}\right)$ be the Zariski open subset of $D \mathscr{X} \times{ }_{s} \mathfrak{X}^{\prime} / S$ which represents the functor $\mathbb{I}:(\mathrm{An} / S)^{\circ} \rightarrow$ Sets defined by $\mathbb{Z}(T)=$ the set of $T$-isomorphisms $\psi: \mathscr{X} \times{ }_{S} T \rightarrow \mathscr{X}^{\prime} \times{ }_{s} T$, where An/S is the category of complex spaces over $S$ (cf. [26]).

We set Aut $\mathscr{X} / S=\operatorname{Isom}_{S}(\mathscr{X}, \mathscr{X})$. Aut $\mathscr{X} / S$ has the natural structure of a complex Lie group over $S$ with a natural relative action on $\mathscr{X}$. In the $a b$ solute case, i.e., when $S$ is a point, Aut $\mathscr{X} / S$ reduces to the usual complex $\mathbb{L i e}$ group Aut $X$ of biholomorphic automorphisms of $X=\mathscr{X}$. We denote by Aut $_{0} X$ the identity component of Aut $X$.

c) Let $(f: \mathscr{X} \rightarrow S, \widetilde{\omega})$ and $\left(f^{\prime}: \mathscr{X}^{\prime} \rightarrow S, \widetilde{\omega}^{\prime}\right)$ be polarized families of compact Kähler manifolds. Then define the functor $\mathbb{I}^{\omega}:(\mathrm{An} / S)^{\circ} \rightarrow$ Sets by

$\mathbb{I}^{\omega}(T)=$ the set of isomorphisms of the pull-backs $\left(f_{T}, \widetilde{\omega}_{T}\right)$ and $\left(f_{T}^{\prime}, \widetilde{\omega}_{T}^{\prime}\right)$ as polarized families.

Then $\mathbb{I}^{\omega}$ is represented by an open and closed subset $\operatorname{Isom}_{s}\left((\mathscr{X}, \widetilde{\omega}),\left(\mathscr{X}^{\prime}, \widetilde{\omega}^{\prime}\right)\right)$ of $\operatorname{Isom}_{s}\left(\mathfrak{X}, \mathfrak{X}^{\prime}\right)$ (cf. $[10,3.2]$ up to a change of notation). In particular the closure $I^{-}$of $\operatorname{Isom}_{S}\left((\mathfrak{X}, \widetilde{\omega}),\left(\mathscr{X}^{\prime}, \widetilde{\omega}^{\prime}\right)\right)$ in $D \mathscr{X} \times{ }_{S} \mathfrak{X}^{\prime} / S$ is analytic in $D \mathscr{X} \times{ }_{S} \mathfrak{X}^{\prime} / S$.

In the absolute case we use the notations $\operatorname{Isom}\left((X, \omega),\left(X^{\prime}, \omega^{\prime}\right)\right), \operatorname{Aut}(X, \omega)$ etc. For instance

$$
\operatorname{Aut}(X, \omega)=\left\{g \in \text { Aut } X ; g^{*} \omega=\omega\right\} .
$$

(2.2) (Grothendieck's criterion for smoothness [16, IV, Th. 3.1]). Let $f$ : $\mathfrak{X} \rightarrow S$ be a morphism of complex spaces. Let $x \in \mathscr{X}$ and $s=f(x)$. Let $A$ be a local $\mathbb{C}$-algebra which is a finite $\mathcal{O}_{S, s}$-algebra. Let $m$ be the maximal ideal of $A$ and $I$ an ideal of $A$ with $m I=0$. Let $S_{1}=$ Specan $A$ and $S_{2}$ the subspace of $S_{1}$ defined by $I$. Let $v_{2}: S_{2} \rightarrow \mathfrak{X}$ be an $S$-morphism with $v_{2}(t)=x$, where $t$ is the unique point of $S_{1}$. Then $f$ is smooth at $x$ if and only if for any $S_{1}$, $S_{2}, v_{2}$ as above we can always find an extension $v_{1}: S_{1} \rightarrow \mathscr{X}$ of $v_{2}$. 
(2.3) The next result is due to Deligne [3].

Proposition 1. Let $f: \mathcal{X} \rightarrow S$ be a proper smooth morphism of complex spaces with connected fibers. Suppose that $X_{o}$ is Kähler for some $o \in \mathbb{S}$. Then for any $i \geqq 0$ and any morphism $\nu: T \rightarrow S$ of complex spaces the natural homomorphism $\nu^{*} R^{i} f_{*} \Theta_{\mathscr{X}} \rightarrow \mathbb{R}^{i} f_{T^{*}} \mathcal{O}_{\mathscr{X}_{T}}$ is isomorphic in a neighborhood of $\nu^{-1}(o)$. Moreover $\mathbb{R}^{i} f_{*} \mathcal{O}_{\mathfrak{X}}$ is free in a neighborhood of $o$.

Proof. See [3, Th. 5.5], where in the proof we may refer to [1, III, Cor. 3.10 and Th. 4.1] instead of (7.8.5) and (6.10.5) of EGA III, respectively.

Since $\nu^{*} R^{2} f_{*} \mathbb{R} \rightarrow R^{2} f_{T^{*}} \mathbb{R}$ is isomorphic, it follows that the commutative diagram

$$
\begin{gathered}
\nu^{*} R^{2} f_{*} \mathbb{R}^{\nu^{*}\left(\eta_{f}\right)} \longrightarrow \nu^{*} R^{2} f_{*} \Theta_{\mathscr{X}} \\
\qquad \downarrow \\
R^{2} f_{T^{*}} \mathbb{R} \underset{\eta_{f_{T}}}{\longrightarrow} \mathbb{R}^{2} f_{T^{*}} \Theta_{\mathscr{X}_{T}}
\end{gathered}
$$

gives the natural identification of $\nu^{*}\left(\eta_{f}\right)$ and $\eta_{f_{T}}$, where $\eta_{f}$ is as in (1.1).

(2.4) Let $\alpha: Z \rightarrow S$ be a morphism of complex spaces. Suppose that $Z$ is a dense Zariski open subset of another complex space $\bar{Z}$ and $\alpha$ extends to a proper morphism $\bar{\alpha}: \bar{Z} \rightarrow S$. Then there exists a unique maximal Zariski open subset $U \subseteq S$ such that $\alpha$ is proper over $U$; in fact, we have only to set $U=S$ $-\bar{\alpha}(\bar{Z}-Z)$. In particular if $Z_{s}=(\bar{Z})_{s}$ for some point $s \in S$, then $s \in U$ and $\alpha$ is proper over some neighborhood of $s$.

\section{§3。 A Kählerian Amalogue of a Theorem of Matsusaka-Mumford}

In this section we prove a refinement of the kählerian analogue of a theorem of Matsusaka-Mumford [22] given in [9].

(3.1) Let $X$ be a compact complex manifold. Then we shall denote a (local) deformation of $X$ by the triple $f: \mathscr{X} \rightarrow S, X_{o}=X, o \in S$, where $f$ is a proper smooth morphism and we always consider $S$ as a germ of a complex space at $o$, or one of its representatives. In particular we have the natural isomorphisms

(*) $H^{2}(\mathfrak{X}, \mathbb{R}) \cong \Gamma\left(S, R^{2} f_{*} \mathbb{R}\right) \cong H^{2}(X, \mathbb{R})$.

Let $(f: \mathscr{X} \rightarrow S, \widetilde{\omega})$ be a polarized family of compact $\mathbb{K a ̈ h l e r}$ manifolds. Then it is expected that for any point $s \in S$ there exist a neighborhood $U$ of $s$ and a Kähler form $\beta$ on $\mathscr{X}_{U}$ which induces $\widetilde{\omega}$ in $\Gamma\left(U, R^{2} f_{U^{*}} \mathbb{R}\right)$. We shall show 
that this is the case when $S$ is nonsingular and of dimension 1 and note that this is even true when $\operatorname{dim} S>1$ (cf. Proposition 5 and Remark 2 below). But for the moment the next lemma is enough for our purposes.

Lemma 1. Let $(X, \omega)$ be a polarized compact Kähler manifold. Let $f$ : $\mathscr{X} \rightarrow S, X_{0}=X, o \in S$, be a deformation of $X$ with $S$ nonsingular. Let $\Omega \in$ $H^{2}(\mathscr{X}, \mathbb{R})$ and $\widetilde{\omega} \in \Gamma\left(S, R^{2} f_{*} \mathbb{R}\right)$ correspond to $\omega$ with respect to the isomorphism (*). Then the following conditions are equivalent. 1) $\eta_{f}(\widetilde{\omega})=0$ in $\Gamma\left(S, R^{2} f_{*} \mathcal{O}_{\mathfrak{X}}\right)$, where $\eta_{f}: \Gamma\left(S, R^{2} f_{*} \mathbb{R}\right) \rightarrow \Gamma\left(S, R^{2} f_{*} \mathcal{O}_{\mathfrak{X}}\right)$ is the natural homomorphism, 2) the restriction $\widetilde{\omega}_{s} \in H^{2}\left(X_{s}, \mathbb{R}\right)$ of $\widetilde{\omega}$ to $X_{s}$ is a Kähler class and 3$) \Omega$ is represented by a real closed $C^{\infty} 2$-form $\beta$ on $\mathcal{X}$ which is a Kähler form when restricted to each fiber of $f$.

Proof. The implication 3) $\rightarrow 2$ ) is obvious. By virtue of (2.3) 1) is equivalent to the condition that $\widetilde{\omega}_{s}$ is of type $(1,1)$ when restricted to each fiber of $f$. From this, the implication 2) $\rightarrow$ 1) follows. We show that 1) implies 3). Fix a Kähler form $\alpha$ on $X$ representing the class $\omega$. Then by Kodaira-Spencer (cf. [18]) there exists a family $\left\{\alpha_{s}\right\}_{s \in S}$ of Kähler forms on $X_{s}$ with $\alpha_{o}=\alpha$ which depends differentiably on $s$. Since $\alpha_{s}$ are harmonic forms with respect to the metrics $g_{s}$ associated to $\alpha_{s}$, by [28, Lemma, p. 196] there exists a real closed $C^{\infty} 2$-form $\beta$ on $\mathcal{X}$ such that $\beta_{o}=\alpha$ and that $\beta_{s}$ is a harmonic form with respect to $g_{s}$. Clearly $\beta$ represents the class $\Omega$. Then since for each $s \in S, \Omega_{s}$ (the restriction of $\Omega$ to $X_{s}$ ) is of type $(1,1)$ as a cohomology class by our assumption and since $\beta_{s}$ is harmonic, $\beta_{s}$ is actually a $(1,1)$-form on $X_{s}$. Moreover (if $s$ is sufficiently near to $o$ ) $\beta_{s}$ are positive forms on $X_{s}$ since so is $\beta_{o}$. Thus $\beta_{s}$ is a $\mathbb{K a ̈ h l e r}$ form on each $X_{s}$ as desired.

q.e.d.

(3.2) Using Lemma 1 we show the next proposition by reducing to an analogous one established in [10].

Proposition 2. Let $\left(f_{i}: \mathfrak{X}_{i} \rightarrow S, \widetilde{\omega}_{i}\right), i=1,2$, be polarized families of compact Kähler manifolds. Then $\mathbb{I}^{-}:=\operatorname{Isom}_{S}\left(\left(\mathscr{X}_{1}, \widetilde{\omega}_{1}\right),\left(\mathscr{X}_{2}, \widetilde{\omega}_{2}\right)\right)^{-}$is proper over $S$, where $I^{-}$is the closure of $\operatorname{Isom}_{S}\left(\left(\mathfrak{X}_{1}, \widetilde{\omega}_{1}\right),\left(\mathfrak{X}_{2}, \widetilde{\omega}_{2}\right)\right)$ in $D \mathfrak{X}_{1} \times_{s} \mathfrak{X}_{2} / S$ (cf. (2.1)).

Proof. Since the problem is topological we may assume that $S$ is reduced. Let $\pi: \hat{S} \rightarrow S$ be a resolution of $S$. Let $\left(\hat{f}_{i}: \hat{\mathscr{X}}_{i} \rightarrow \hat{S}, \hat{\omega}_{i}\right)$ be the pull-back of $\left(f_{i}, \widetilde{\omega}_{i}\right)$ to $\hat{S}$ by $\pi$. Then with respect to the natural isomorphism $\left(D \mathscr{X}_{1} \times{ }_{S} \mathfrak{X}_{2} / S\right)$ ${ }_{S} \hat{S} \cong D \hat{\mathscr{X}}_{1} \times \hat{S}_{S} \hat{\mathscr{X}}_{2} / \hat{S}, I^{-} \times{ }_{S} \hat{S}$ is naturally considered as a closed analytic subspace of $\operatorname{Isom}_{\hat{S}}\left(\left(\hat{\mathfrak{X}}_{1}, \hat{\omega}_{1}\right),\left(\hat{\mathcal{X}}_{2}, \hat{\omega}_{2}\right)\right)^{-}$(in fact they coincide). Hence it suffices to show the properness for $\left(\hat{f}_{i}, \hat{\omega}_{i}\right)$. So we may assume from the beginning that $S$ is 
nonsingular. Then since the problem is local on $S$, by Lemma 1,2$) \rightarrow 3$ ) we may assume that there exists a real $d$-closed $\mathbb{C}^{\infty} 2$-form $\beta_{i}$ on $\mathscr{X}_{i}$ such that $\left(\beta_{i}\right)_{s}$ is a Kähler form and is a representative of the class $\left(\widetilde{\omega}_{i}\right)_{s}$ for each $s \in S$. Then the proposition follows from Proposition 3 of [10].

q.e.d.

For later reference we record a special case where $S$ is a point and where $\mathscr{X}_{1}=\mathscr{X}_{2}$ and $\widetilde{\omega}_{1}=\widetilde{\omega}_{2}$.

Corollary [6]. For any polarized compact Kähler manifold $(X, \omega), \operatorname{Aut}(X, \omega)$ has only a finite number of connected components.

(3.3) Let $D=\{t \in \mathbb{C} ;|t|<1\}$ be the unit disc. Let $D^{\prime}=D-\{0\}$. Let $\left(f_{i}\right.$ : $\left.\mathscr{X}_{i} \rightarrow D, \widetilde{\omega}_{i}\right), i=1,2$, be polarized families of compact Kähler manifolds over $D$. The next proposition is a kählerian analogue of Theorem 2 of MatsusakaMumford [22], a little weaker version of which was established in [9].

Proposition 3. Let $\varphi: \mathscr{X}_{1} \rightarrow \mathscr{X}_{2}$ be a bimeromorphic map over $D$ which induces over $D^{\prime}$ an isomorphism of the induced polarized families $\left(f_{1, D^{\prime}}, \widetilde{\omega}_{1, D^{\prime}}\right)$ and $\left(f_{2, D^{\prime}}, \widetilde{\omega}_{2, D^{\prime}}\right)$. Then if $X_{1,0}$ is not ruled, $\varphi$ must be isomorphic.

Here a compact complex manifold $X$ is said to be ruled if there exist a compact complex manifold $Y$ and a holomorphic vector bundle $E$ on $Y$ of rank $\geqq 2$ such that $X$ is bimeromorphic to the associated projective bundle $\mathbb{P}(E)$.

Before the proof we first derive from this proposition the following:

Proposition 4. Let $\left(f_{i}: \mathfrak{X}_{i} \rightarrow S, \widetilde{\omega}_{i}\right), i=1,2$, be polarized families of compact Kähler manifolds with $X_{1, o}$ non-ruled for some $o \in S$. Then $I=\operatorname{Isom}_{S}\left(\left(X_{1}\right.\right.$, $\left.\left.\widetilde{\omega}_{1}\right),\left(\mathfrak{X}_{2}, \widetilde{\omega}_{2}\right)\right)$ is proper over some neighborhood of o in $S$.

Proof. Since $I^{-}=\operatorname{Isom}_{S}\left(\left(\mathfrak{X}_{1}, \widetilde{\omega}_{1}\right),\left(\mathfrak{X}_{2}, \widetilde{\omega}_{2}\right)\right)$ is proper over $S$ by Proposition 2, it suffices to show that $I_{o}^{-}=I_{o}$ (cf. (2.4)). So assuming that $I_{o}^{-} \neq I_{o}$ we shall derive a contradiction. Take a morphism $h: D \rightarrow I^{-}$with $h(0) \in$ $I_{o}^{-}-I_{o}$ and $h^{-1}\left(I_{o}^{-}-I\right)=\{0\}$, where $D=\{t \in \mathbb{C} ;|t|<1\}$. Let $\bar{h}$ be the composition of $h$ with the natural projection $I^{-} \rightarrow S$. Let $\left(\hat{f}_{i}: \hat{\mathcal{X}}_{i} \rightarrow D, \hat{\omega}_{i}\right), i=1,2$, be the pull-back of $\left(f_{i}, \widetilde{\omega}_{i}\right)$ by $\bar{h}$. Then by our choice of $h$ there exists a bimeromorphic $D$-map $\varphi: \hat{\mathscr{X}}_{1} \rightarrow \hat{\mathscr{X}}_{2}$ which is isomorphic over $D^{\prime}=D-\{0\}$, but not isomorphic over the whole $D$ (cf. [10]). Moreover if $\varphi^{\prime}: \hat{\mathscr{X}}_{1}^{\prime} \rightarrow \hat{\mathscr{X}}_{2}^{\prime}$ is the induced isomorphism with $\hat{X}_{i}^{\prime}=\hat{f}_{i}^{-1}\left(D^{\prime}\right)$, then we have $\varphi^{\prime *} \hat{\omega}_{2}=\hat{\omega}_{1}$. It then follows from Proposition 3 that $\varphi$ must be isomorphic, which is a contradiction. q.e.d.

Corollary. Under the above assumptions the set $S_{1}:=\left\{s \in S ;\left(X_{1 s}, \widetilde{\omega}_{1 s}\right) \cong\right.$ 
$\left.\left(X_{2 s}, \widetilde{\omega}_{2 s}\right)\right\}$ is an analytic subset of $S$ in a neighborhood of $o$.

Proof. $S_{1}$ is the image of $\operatorname{Isom}_{S}\left(\left(\mathscr{X}_{1}, \widetilde{\omega}_{1}\right),\left(\mathfrak{X}_{2}, \widetilde{\omega}_{2}\right)\right)$ by a proper map. q.e.d.

(3.4) Let $(X, \omega)$ be a polarized compact Kähler manifold. Then a polarized (local) deformation of $(X, \omega)$ is a polarized family of compact Kähler manifolds $(f: \mathcal{X} \rightarrow S, \widetilde{\omega})$ such that $\left(X_{o}, \widetilde{\omega}_{o}\right)=(X, \omega)$ for a point $o \in S$ with $S$ considered as an analytic germ at $o$.

Now Proposition 3 follows from Theorem 4.3 of [9] if $\widetilde{\omega}_{i}$ is induced from a Kähler class $\Omega_{i}$ on $\mathscr{X}_{i}$. This is indeed guaranteed by the following:

Proposition 5. Let $(X, \omega)$ be a polarized compact Kähler manifold. Let $(f: \mathscr{X} \rightarrow S, \widetilde{\omega}),\left(X, \widetilde{\omega}_{o}\right)=(X, \omega), o \in S$, be a polarized deformation of $(X, \omega)$. Suppose that $\operatorname{dim} S=1$ and $S$ is nonsingular. Then the class $\Omega \in H^{2}(\mathscr{X}, \mathbb{R})$ which corresponds to $\omega$, or $\widetilde{\omega}$, in the isomorphism (*) is a Kähler class.

The proof which follows is suggested by the referee. (The original proof of ours is by way of the harmonic theory of Kodaira-Spencer.)

We proceed in steps. Let $\mathcal{E}_{\mathscr{X}}^{0, q}$ be the sheaf of germs of $C^{\infty}(0, q)$-forms on $\mathscr{X}$ and set $\mathcal{E}_{\mathscr{X}}=\mathcal{E}_{\mathscr{X}}^{0,0}$, the sheaf of germs of $C^{\infty}$ functions on $\mathscr{X}$. Let $t$ be the local parameter of $S$ with center $o$. Let $\mathcal{G}=t \mathcal{O}_{\mathfrak{X}}$ and $\mathcal{g}=(t, \bar{t}) \mathcal{E}_{\mathfrak{X}}$. Let $\hat{\mathcal{O}}_{\mathscr{X}}=\lim \mathcal{O}_{\mathfrak{X}} / \mathcal{I}^{n}$ and $\hat{\mathcal{E}}_{\mathscr{X}}^{0, q}=\lim \mathcal{E}_{\mathscr{X}}^{0, q} / \mathscr{g}^{n} \mathcal{E}_{\mathscr{X}}^{0, q}$. Then the usual $\bar{\partial}$-operator $\mathcal{E}_{\mathscr{X}}^{0, q} \rightarrow$ $\mathcal{E}_{\mathscr{X}}^{0, q+1}$ induces a similar homomorphism $\hat{\mathcal{E}}_{\mathscr{X}}^{0, q} \rightarrow \hat{\mathcal{E}}_{\mathscr{X}}^{0, q+1}$ (still denoted by $\bar{\partial}$ ) with

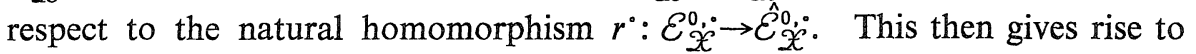
the "formal Dolbeault complex"

(耑) $0 \rightarrow \hat{\mathcal{O}}_{\mathscr{X}} \rightarrow \hat{\mathcal{E}}_{\mathfrak{X}} \rightarrow \hat{\mathcal{E}}_{\mathscr{X}}^{0,1} \rightarrow \cdots \rightarrow \hat{\mathcal{E}}_{\mathscr{X}}^{0, q} \rightarrow \cdots$

which turns out to be a fine resolution of $\hat{\mathcal{O}}_{\mathfrak{X}}$; this is indeed a special case of the formal Dolbeault lemma proved by Bingener [31] and the author [13] independently. As a consequence of this we note the following:

Lemma 2。 The natural homomorphism $H^{q} \Gamma\left(X_{o},\left.\mathcal{E}_{\mathscr{X}}^{0, \cdot}\right|_{X_{0}}\right) \rightarrow H^{q} \Gamma\left(X_{o}, \hat{\mathcal{E}}_{\dot{X}}^{0.0}\right)$ induced by $r^{\circ}$ is injective for any $q \geqq 0$.

Proof. Let $\left(R^{q} f_{*} \mathcal{O}_{\mathfrak{X}}\right)^{\wedge}=\lim R^{q} f_{*} \mathcal{O}_{\mathfrak{X}} / \mathcal{I}^{n} R^{q} f_{*} \mathcal{O}_{\mathfrak{X}}$. Let $\hat{f}: \hat{\mathcal{X}} \rightarrow \hat{S}$ be the

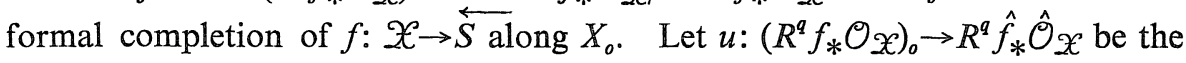
composition of the natural injection $\left(R^{q} f_{*} \mathcal{O}_{\mathscr{X}}\right)_{0} \rightarrow\left(R^{q} f_{*} \mathcal{O}_{\mathscr{X}}\right)^{\wedge}$ and isomorphism $\left(R^{q} f_{*} \mathcal{O}_{\mathfrak{X}}\right)^{\wedge} \rightarrow R^{q} \hat{f}_{*} \hat{\mathcal{O}}_{\mathfrak{X}}$ (cf. [1, VI, 4.5]). The lemma then follows from the commutative diagram 


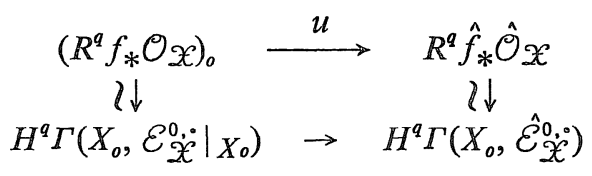

where the vertical arrows are the usual and the formal Dolbeault isomorphisms (induced by (垹) in the formal case).

q.e.d.

Using Lemma 2 we shall next show the following:

Lemma 3. Let $\beta$ be a $\bar{\partial}$-closed $C^{\infty}(0,2)$-form on $\mathfrak{X}$ whose restriction to each fiber of $f$ vanishes identically. Then $\beta=\bar{\partial} \alpha$ for some $C^{\infty}(0,1)$-form $\alpha$ (in a neighborhood of $X_{o}$ ).

Proof. First we note that $\beta$ is written in the form $\beta=\varphi \wedge d \bar{t}$ for some $C^{\infty}$ $(0,1)$-form $\varphi$ on $\mathcal{X}$. Indeed, from the local consideration it is clear that we may write $\beta=\varphi_{1} \wedge d \bar{t}$ for some $\varphi_{1} \in \Gamma\left(X, \mathcal{E}_{\mathscr{X}}^{0} / \mathcal{E}_{\mathfrak{X}} d \vec{t}\right)$ in the obvious sense and then it suffices to take $\varphi$ to be any lift of $\varphi_{1}$ with respect to the natural surjection $\Gamma\left(X, \mathcal{E}_{\mathscr{X}}^{0,1}\right) \rightarrow \Gamma\left(X, \mathcal{E}_{\mathscr{X}}^{0,1} / \mathcal{E}_{\mathscr{X}} d \bar{t}\right)$. Similar remark applies also to any $C^{\infty}$ $(0, q)$-form $\psi, q \geqq 1$, with $d \bar{t} \wedge \psi=0$ and will be used without further mention.

Now we shall show by induction on $n \geqq 1$ the following statement: $(*)_{n}$. There exist $C^{\infty}(0,1)$-forms $\alpha_{n}, \varphi_{n}$ on $\mathscr{X}$ such that $\beta$ is written in the form $\beta=$ $\bar{t}^{n} \bar{\partial} \varphi_{n}+\bar{\partial} \alpha_{n}$ and that $\alpha_{n+1} \equiv \alpha_{n} \bmod g^{n+1}$. First of all, when $n=1$, we have only to take $\varphi_{1}=\varphi$ and $\alpha_{1}=-\varphi \bar{t}$. Suppose next that $(*)_{k}$ are proved for any $1 \leqq k \leqq n$. Then $\bar{\partial} \beta=n \bar{t}^{n-1} d \bar{t} \wedge \bar{\partial} \varphi_{n}=0$, which implies that $\bar{\partial} \varphi_{n}=\psi_{n} \wedge d \bar{t}$ for some $C^{\infty}(0,1)$-form $\psi_{n}$ on $\mathscr{X}$. Then we have

$$
\beta=\bar{t}^{n} d \bar{t}+\bar{\partial} \alpha_{n}=\bar{\partial}\left(\alpha_{n}-1 /(n+1) \bar{t}^{n+1} \psi_{n}\right)+\bar{t}^{n+1} \bar{\partial}\left(1 /(n+1) \psi_{n}\right) .
$$

Hence $\alpha_{n+1}:=\alpha_{n}-1 /(n+1) \bar{t}^{n+1} \psi_{n}$ and $\varphi_{n+1}:=1 /(n+1) \psi_{n}$ satisfy $(*)_{n+1}$. Thus $(*)_{n}$ are true for all $n \geqq 1$. Now $\hat{\alpha}:=\left\{\alpha_{n}\right\}$ determines an element of $\Gamma\left(X_{o}, \hat{\mathcal{E}}_{\mathscr{X}}^{0,1}\right)$ with $\bar{\partial} \hat{\alpha}=\hat{\beta}$, where $\hat{\beta}$ is the natural image of $\beta$ in $\Gamma\left(X_{o}, \hat{\mathcal{E}}_{\mathscr{X}}^{0,1}\right)$. The lemma thus follows from Lemma 2.

q.e.d.

Proof of Proposition 5. We have to show that $\Omega$ is represented by a Kähler form. By Lemma 1 there exists a real $d$-closed $C^{\infty} 2$-form $\beta$ representing $\Omega$ such that when restricted to each fiber it is a Kähler form and hence in particular of type $(1,1)$. Let $\beta=\beta^{2,0}+\beta^{1,1}+\beta^{0,2}$ be the type decomposition of $\beta$, where $\beta^{0,2}=\bar{\beta}^{2,0}$ and $\bar{\partial} \beta^{0,2}=0$. Moreover $\beta^{0,2}$ restricted to each fiber vanishes. Hence by Lemma 2 we may write $\beta^{0,2}=\bar{\partial} \alpha$ for some $C^{\infty}(0,1)$-form $\alpha$ on $\mathscr{X}$. Then $\beta^{\prime}:=\beta-d(\alpha+\bar{\alpha})$ is a $d$-closed $(1,1)$-form representing $\Omega$. Moreover $\beta_{s}^{1,1}=\beta_{s}^{\prime}$ for each $s$. Thus $\beta^{\prime}$ gives a Kähler form on each fiber. Finally 
replacing $\beta^{\prime}$ by $\beta^{\prime}+N d t \wedge d \bar{t}$ for some sufficiently large constant $N>0$ we get a Kähler form representing $\Omega$.

Remark 2. As pointed out by the referee the above proof also works for the case $\operatorname{dim} S>1$ (with $S$ nonsingular) by a suitable modification of the proof of Lemma 3.

\section{§4. Cohomological Flatmess of the Relative Tangent Sheaf}

In this section we shall show the cohomological flatness of $\Theta \mathscr{X} / S$ for any polarized family $(f: \mathfrak{X} \rightarrow S, \widetilde{\omega})$ of compact Kähler manifolds such that $\mathrm{Aut}_{0} X_{s}$ is a complex torus for any $s \in S$, where $\Theta \mathscr{X} / S$ is the relative tangent sheaf associated to $f$, i.e., the sheaf of germs of holomorphic vector fields on $\mathscr{X}$ which are tangent to the fibers of $f$.

(4.1) We begin with a general definition. Let $f: \mathscr{X} \rightarrow S$ be a morphism of complex spaces and $\mathscr{F}$ an $f$-flat coherent analytic sheaf on $\mathscr{X}$. Then $\mathscr{F}$ is said to be cohomologically flat (in dimension zero) at $s \in S$ with respect to $f$, if the following equivalent conditions are satisfied (cf. [1, III, Cor. 3.7]):

i) For any morphism $\nu: T \rightarrow S$ of complex spaces, the natural map $\nu^{*} f_{*} \mathscr{I}$ $\rightarrow f_{T^{*}} \tilde{\nu} * \mathscr{F}$ is isomorphic at any point $t \in T$ with $\nu(t)=s$, where $\tilde{\nu}: \mathcal{X} \times{ }_{S} T \rightarrow \mathscr{X}$ is the natural morphism.

ii) Let $m$ be the maximal ideal of $\mathcal{O}_{s}$ at $s$. Then the restriction maps $f_{*}\left(\mathscr{F} / m^{k} \mathscr{F}\right)_{s} \rightarrow H^{0}\left(X_{s}, \mathscr{F} / m \mathscr{F}\right)$ are surjective for all $k>0$.

Moreover in this case $f_{*} \mathscr{F}$ is free in a neighborhood of $s$. Further, when $S$ is reduced, $\mathscr{F}$ is cohomologically flat at $s$ if and only if $d(s):=\operatorname{dim} H^{0}\left(X_{s}, \mathscr{F} / m \mathscr{F}^{1}\right)$, $s \in S$, is constant in a neighborhood of $s$ (cf. [1, Th. 4.12]). We say that $\mathscr{F}$ is cohomologically flat with respect to $f$ if so is $\mathscr{F}$ at any point of $S$. Clearly, if $\mathscr{F}$ is cohomologically flat with respect to $f$, then $\tilde{\nu}^{*} \mathscr{F}$ is cohomologically flat with respect to $f_{T}$, where $\tilde{\nu}$ is as in i) above.

Now the purpose of this section is to prove the following:

Theorem $\mathbb{1}$. Let $X$ be a compact connected Kähler manifold. Let $f: \mathscr{X} \rightarrow S$, $X_{o}=X, o \in S$, be a deformation of $X$. Suppose that Aut $_{0} X$ is a complex torus. ${ }^{*}$ Then the relative tangent sheaf $\Theta \mathscr{X} / S$ is cohomologically flat (in dimension zero) at $o$. In particular $\operatorname{dim} H^{0}\left(X_{s}, \Theta_{X_{s}}\right)$ is independent of $s$ in a neighborhood of $o$.

Remark 3. The last assertion is shown first by Matsusaka under the assumption that there exists a relatively ample line bundle $L$ on $\mathscr{X}$ and that

*) The case where $\mathrm{Aut}_{0} X$ reduces to the identity is included. 
$\left(X, c_{1}^{R}\left(L_{o}\right)\right)$ is in $\mathfrak{B}$ (cf. $\S 6$, below), where $c_{1}^{R}\left(L_{o}\right)$ is the real chern class of $L_{o}$. (See Corollary to Proposition 11 of [21].)

(4.2) First we note that for a proper smooth morphism $f: \mathscr{X} \rightarrow S$ the cohomological flatness of $\Theta \mathfrak{X} / S$ has the following significance.

Proposition 6. Let $f: \mathscr{X} \rightarrow S$ be a proper smooth morphism of complex spaces. Let $s$ be a fixed point of $S$. Then the following conditions are equivalent.

1) $\Theta \mathscr{X} / S$ is cohomologically flat at $s$ with respect to $f$.

2) $\alpha$ : Aut $\mathscr{X} / S \rightarrow S$ is smooth at any point of $\mathrm{Aut}_{0} X_{s}$ with respect to the natural inclusion Aut $_{0} X_{s} \subseteq(\text { Aut } \mathscr{X} / S)_{s}$.

We first prove a lemma.

Lemma 4. $\alpha$ is smooth at any point of $\mathrm{Aut}_{0} X$ if it is smooth at $e(s)$, where $e$ is the identity section of $\alpha$.

Proof. Let $a \in \mathrm{Aut}_{0} X_{s}$ be arbitrary. We first show that there exists a holomorphic section $\varepsilon: S \rightarrow$ Aut $\mathscr{X} / S$ with $\varepsilon(s)=a$. Let $U$ be a neighborhood of $e(s)$ in Aut $\mathscr{X} / S$ such that $\alpha$ is smooth on $U$. Then we can find an integer $m>0$ such that the image of $U_{S}^{m}:=U \times{ }_{S} \cdots \times{ }_{S} U$ ( $m$-times) by the natural $S$ morphism $\pi: U_{S}^{m} \rightarrow$ Aut $\mathscr{X} / S$ (induced by the relative multiplication of Aut $\mathscr{X} / S$ ) contains $a$. Take any point $u \in U_{S}^{m}$ with $\pi(u)=a$. Since $U_{S}^{m} \rightarrow S$ is smooth, we can find a holomorphic section $\varepsilon^{m}: S \rightarrow U_{S}^{m}$ with $\varepsilon^{m}(s)=u$. Let $\varepsilon=\pi \varepsilon^{m}$ : $S \rightarrow$ Aut $\mathscr{X} / S$. Then $\varepsilon$ is a desired holomorphic section with $\varepsilon(s)=a$. $\varepsilon(S)$ then defines by translation an $S$-automorphism $\varepsilon^{*}$ of Aut $\mathfrak{X} / S$ as a complex space over $S$ such that $\varepsilon^{*}(e(s))=a$. The lemma follows.

Proof of Proposition 6. (cf. Wavrik [29]) 1) $\rightarrow 2$ ). Suppose that $\Theta_{\mathfrak{X} / S}$ is cohomologically flat. We shall show that $\alpha$ is smooth. By Lemma 4 we have only to check this at $e(s)$. We use Grothendieck's criterion (2.2): in the notation there for a given $S$-morphism $v_{2}: S_{2} \rightarrow$ Aut $\mathscr{X} / S$ with $v_{2}(t)=e(s)$, we want to find its extension $v_{1}: S_{1} \rightarrow$ Aut $\mathscr{X} / S$. Let $\mathscr{X}_{i}:=\mathscr{X} \times{ }_{s} S_{i}$ and $e_{i}$ : $S_{i} \rightarrow$ Aut $\mathscr{X}_{i} / S_{i}$ the identity section, $i=1,2$. Let $m_{i}$ be the maximal ideal of $S_{i}$ at $t$. Note the isomorphism Aut $\mathscr{X} / S \times{ }_{s} S_{i} \cong$ Aut $\mathscr{X}_{i} / S_{i}$. Then $v_{2}$ corresponds to a holomorphic section $v_{2}^{\prime} \in \Gamma\left(S_{2}\right.$, Aut $\left.\mathscr{X}_{2} / S_{2}\right)$ with $v_{2}^{\prime}(t)=e_{2}(t)$.

Now we have the natural isomorphism of sets $\Gamma_{e}\left(S_{i}\right.$, Aut $\left.\mathscr{X}_{i} / S_{i}\right) \cong H^{0}\left(\mathscr{X}_{i}\right.$, $\left.m_{i} \Theta \mathscr{X}_{i} / S_{i}\right)$, where $\Gamma_{e}\left(S_{i}\right.$, Aut $\left.\mathscr{X}_{i} / S_{i}\right)=\left\{\psi \in \Gamma\left(S_{i}\right.\right.$, Aut $\left.\left.\mathscr{X}_{i} / S_{i}\right) ; \psi(t)=e_{i}(t)\right\}$ (cf. [29, $\S 3])$. On the other hand, by the cohomological flatness of $\Theta \mathscr{X} / S$ the natural morphism $H^{0}\left(\mathscr{X}_{1}, m_{1} \Theta \mathfrak{X}_{1} / S_{1}\right) \rightarrow H^{0}\left(\mathfrak{X}_{2}, m_{2} \Theta \mathfrak{X}_{2} / S_{2}\right)$ is surjective. Hence we can 
find an extension $v_{1}^{\prime} \in \mathbb{\Gamma}_{e}\left(\mathbb{S}_{1}\right.$, Aut $\left.\mathfrak{X}_{1} / S_{1}\right)$ of $v_{2}^{\prime}$, which in turn gives a desired extension $v_{1}$ of $v_{2}$.

$2) \rightarrow 1$ ). (cf. [29, Prop. 4.3]) Suppose that $\Theta \mathscr{X} / S$ is not cohomologically flat. We shall show that $\alpha$ is not smooth at $e(s)$. Let $m$ be the maximal ideal of $S$ at $s$. By our assumption there exist an integer $k>0$ and $\theta \in H^{0}\left(X_{s}, \Theta_{X_{s}}\right)$ which is not in the image of the natural homomorphism $f_{*}\left(\Theta \mathscr{X} / S / m^{k} \Theta \mathscr{X} / S\right) \rightarrow H^{0}\left(X_{s}, \Theta_{X_{s}}\right)$. Replacing $S$ by the subspace defined by $m^{k}$ we may assume that $m^{k}=0$ on $S$. Let $T=S \times \operatorname{Spec} \mathbb{C}[\varepsilon]$ and $T^{\prime}=\{s\} \times \operatorname{Spec} \mathbb{C}[\varepsilon]$, where $\varepsilon$ is the dual number; $\varepsilon^{2}=0$. Consider $T$ as a complex space over $S$ via the natural projection $T$ $\rightarrow S$. Let $o \in \operatorname{Spec} \mathbb{C}[\varepsilon]$ be the unique point and $n$ the maximal ideal of $T$ at $(s, o)$. Then $\varepsilon \theta$ is naturally considered as a section of $f_{T^{\prime *}} \Theta_{X_{T^{\prime}} / T^{\prime}}$ and it is not in the image of the natural map $f_{T^{*}} \Theta \mathfrak{X}_{T} / T \rightarrow f_{T^{\prime * n}} \Theta \mathfrak{X}_{T^{\prime *} / T^{\prime}}$. Since, in the same notation as above, $f_{Y^{*}}\left(n \Theta \mathscr{X}_{Y} / Y\right)_{(s, o)} \cong \Gamma_{e}\left(Y\right.$, Aut $\left.\mathfrak{X}_{Y} / Y\right)\left(Y=T, T^{\prime}\right)$ by [29], this implies that $\alpha$ is not smooth at $e(s)$ by (2.2).

q.e.d.

(4.3) In general let $S$ be any Artin space, i.e., a complex space with a unique point, say $o$. Let $f: \mathscr{X} \rightarrow S$ be a proper smooth morphism with connected fibers. Set $X=X_{0}$. Since $S$ is an Artin space, Aut $\mathscr{X}$ and Aut $S$ have the natural structure of complex $\mathbb{L i e}$ groups (cf. [4]). Moreover $f$ induces the natural homomorphism Aut $\mathscr{X} \rightarrow$ Aut $S$, and hence Aut $_{0} \mathfrak{X} \rightarrow$ Aut $_{0} S$. (Consider for instance $S$ as a distinguished connected component of the Douady space of $\mathscr{X}$ (cf. [17, Lemma 3]) and consider the induced action of Aut $\mathscr{X}$ on S.) Denote the last homomorphism by $f_{*}$. Let $G$ and $N$ be the image and the kernel of $f_{*}$ respectively. Let $u:$ Aut $_{0} \mathscr{X} \rightarrow \mathrm{Aut}_{0} X$ be the natural restriction homomorphism to $X=\mathscr{X}_{\text {red }}$. Then by associating to any $g \in G$ the coset of $u\left(f_{*}^{-1}(g)\right)$ we get a map $w: G \rightarrow E$ of $G$ into the coset space $E:=\operatorname{Aut}_{0} X / u(N)$. It is easy to see that $u(N)$ is closed in $\mathrm{Aut}_{0} X$ and $w$ is holomorphic.

Lemma 5. Suppose that $X$ is Kähler and that Aut $_{0} X$ is a complex torus. Then $w$ is a constant map.

First we recall some terminology from [6]. (See [6] for the more detail.) A meromorphic Lie group is a complex Lie group with a bimeromorphic equivalence class of its compactifications. An algebraic group is a special case of meromorphic Lie groups if we consider its algebraic compactifications as the corresponding equivalence class of compactifications. A homomorphism $G_{1} \rightarrow G_{2}$ of meromorphic $\mathbb{L i e}$ groups is said to be meromorphic if it extends to a meromorphic map $G_{1}^{*} \rightarrow G_{2}^{*}$ between any compactifications $G_{i}^{*}$ of $G_{i}$ in the equivalence classes. 
Proof of Lemma 5. Since $X$ is Kähler, Aut $\mathfrak{X}$ has the natural structure of a meromorphic Lie group (cf. [5, Th. 5.3], [6, Th. 3.5]). (See also Corollary to Theorem of [11], where a gap in the argument in [5] is filled.) On the other hand, since $S$ is an Artin space, Aut $S$ has the natural structure of a linear algebraic group, e.g., as a closed subgroup of $G L(A)$, where $A:=\mathcal{O}_{S, o}$ is considered a finite dimensional $\mathbb{C}$-vector space. Then by [6, Lemma 2.4], with respect to these meromorphic Lie group structures $f_{*}$ is a meromorphic homomorphism. In particular the image $G$ of $f_{*}$ is a linear algebraic subgroup of Aut $S$. Moreover by the standard arguments we see readily that for any algebraic compactification $G^{*}$ of $G, w$ extends to a meromorphic map $w^{*}: G^{*} \rightarrow \mathbb{E}$. It follows then that $w$ is a constant map since $E$ is a complex torus and $G$ is linear algebraic (cf. [6, Lemma 3.8]). q.e.d.

(4.4) Let $X$ be a compact complex manifold. Let $f: \mathcal{X} \rightarrow S, X_{0}=X, o \in S_{\text {, }}$ be the Kuranishi family of $X[19]$. Let $m$ be the maximal ideal of $\mathcal{O}_{S}$ at $o$. Let $S_{n}$ be the subspace of $S$ defined by $m^{n+1}$. Let $\mathfrak{X}_{n}=\mathscr{X}_{S_{S}} S_{n}$. Let $f_{n}: \mathscr{X}_{n} \rightarrow$ $S_{n}$ be the induced morphism.

Lemma 6. The restriction homomorphism $u_{n}$ : Aut $\mathfrak{X}_{n} \rightarrow$ Aut $X$ is surjective for any $n \geqq 0$.

Proof. Take any $h \in$ Aut $X$. The versality of $\mathbb{K}$ uranishi family implies that there exist morphisms $\tilde{h}: \mathscr{X}_{n} \rightarrow \mathcal{X}_{n}$ and $\bar{h}: S_{n} \rightarrow S_{n}$ such that $\left.\tilde{h}\right|_{X_{0}=X}=h$ and that the following diagram is cartesian

$$
\begin{array}{cc}
\mathscr{\chi}_{n} \stackrel{\tilde{h}}{\longrightarrow} \mathscr{X}_{n} \\
f_{n} \downarrow \\
\mathbb{S}_{n} \stackrel{\bar{h}}{\longrightarrow}{ }^{\downarrow} \mathbb{S}_{n} .
\end{array}
$$

Thus it suffices to check that $\bar{h}$, and hence $\tilde{h}$ also, is an automorphism. Since $S_{n}$ is an Artin space, this follows if we see that $\bar{h}$ is an embedding, or the differential $\bar{h}_{*}$ of $\bar{h}$ is injective. In fact, if $\bar{h}_{*}$ is not injective, there is an embedding $\iota: S_{\varepsilon}=$ Spec $\mathbb{C}[\varepsilon] \rightarrow S_{n}$ such that $\mathscr{X}_{n} \times{ }_{S_{n}} S_{\varepsilon} \rightarrow S_{\varepsilon}$ is a trivial family, contradicting the fact that $f$ is the Kuranishi family, where $\mathbb{C}[\varepsilon], \varepsilon^{2}=0$, is the ring of dual numbers.

q.e.d.

Proof of Theorem 1. Clearly we may assume that $f$ is the $\mathbb{K}$ uranishi family of $X$. Further by the (second) definition of the cohomological flatness it suffices then to show the theorem for each $f_{n}$ in the notation above. Let Aut $_{0} \mathcal{X}_{n} / S_{n}$ be the connected component of Aut $\mathscr{X}_{n} / S_{n}$ containing Aut $X_{\text {. Then }}$ by Prop- 
osition 6 we have only to show the smoothness of Aut $_{0} \mathscr{X}_{n} / S_{n}$ over $S_{n}$. First, by Lemmas 5 and 6 we see immediately that $\operatorname{Aut}_{0} X=u_{n}\left(N_{n}\right)$, where $N_{n}$ is the kernel of $f_{n} \mid$ Aut $_{0} \mathscr{X}_{n}$. By definition $N_{n}$ is just the group of automorphisms of $\mathscr{X}_{n}$ over $S_{n}$ inducing those of $\mathrm{Aut}_{0} X$ on $X$, or in other words, the group of holomorphic sections of $\alpha_{n}$ : Aut $_{0} \mathscr{X}_{n} / S_{n} \rightarrow S_{n}$. Thus for any $a \in \mathrm{Aut}_{0} X$ if we take a local holomorphic section $v$ of $N_{n} \rightarrow$ Aut $_{0} X$ defined in a neighborhood $V$ of $a, v$ defines a holomorphic family of sections of $\alpha_{n}$ parametrized by $V \subseteq \mathrm{Aut}_{0} X$ $=\left(\text { Aut }_{0} \mathscr{X}_{n} / S_{n}\right)_{o}$, which in turn gives an $S_{n}$-isomorphism of a neighborhood of $a$ in Aut $_{0} \mathfrak{X}_{n} / S_{n}$ to $S_{n} \times V$. Thus $\alpha_{n}$ is smooth.

q.e.d.

Remark 4. Pushing the above arguments a little further, we can show more generally the following: Let $f: \mathcal{X} \rightarrow S, X_{o}=X, o \in S$, be a deformation of a compact connected Kähler manifold $X$. Then $\operatorname{dim} T\left(X_{s}\right)$ is lower semicontinuous in a neighborhood of $o$, where $T\left(X_{s}\right)=T\left(\mathrm{Aut}_{0} X_{s}\right)$ is the Albanese torus of $\mathrm{Aut}_{0} X_{s}$ (cf. [6]). The proof will be given elsewhere.

(4.5) Since Theorem 1 is important, we shall give an alternative proof in the following special case using the notion of polarized Kuranishi family (to be defined in $§ 5$ ).

Proposition 7. Let $(f: \mathcal{X} \rightarrow S, \widetilde{\omega})$ be any polarized family of compact Kähler manifolds with $S$ reduced. Suppose that $\mathrm{Aut}_{0} X_{0}$ is a complex torus for some $o \in S$. Then $\Theta_{\mathfrak{X} / S}$ is cohomologically flat at $o$.

We first prove a lemma. Let $(X, \omega)$ be a polarized compact Kähler manifold in general. Let $(f: \mathscr{X} \rightarrow T, \widetilde{\omega}),\left(X_{o}, \widetilde{\omega}_{o}\right)=(X, \omega), o \in T$, be the Kuranishi family of $(X, \omega)$ (cf. Remark 6 in $\S 5$ ). Let $r_{i}: T \times T \rightarrow T$ be the projection to the $i$-th factor. Let $\left(f_{i}: \mathscr{X}_{i} \rightarrow T \times T, \widetilde{\omega}_{i}\right), i=1,2$, be the pull-back of $(f, \widetilde{\omega})$ via $r_{i}$. Let $I=\operatorname{Isom}_{T \times T}\left(\left(\mathscr{X}_{1}, \widetilde{\omega}_{1}\right),\left(\mathscr{X}_{2}, \widetilde{\omega}_{2}\right)\right)$ and let $r: I \rightarrow T \times T$ be the natural morphism. Let $J$ be the connected component of $I$ containing Aut $X$ with respect to the natural identification of $\operatorname{Aut}(X, \omega)$ with $I_{(o o)}$. Let $Q=r(J)$.

Lemma 7. If Aut $_{0} X$ is a complex torus, then $Q$ is an analytic subset of $T \times T$ and $\left.r_{i}\right|_{Q}: Q \rightarrow T$ is a finite morphism.

Proof. Since $J_{(o, 0)}=\mathrm{Aut}_{0} X$, by (2.4) $\gamma_{J}=\gamma_{\mid J}: J \rightarrow T \times T$ is proper (if we restrict $T$ ). Hence $Q$ is analytic. Then for the finiteness of $r_{i \mid Q}$ it suffices to show that $Q_{o}:=r_{i}^{-1}(o) \cap Q_{\text {red }}$ reduces to the point $(o, o)$. Consider $Q_{o}$ as a subspace of $T=r_{i}^{-1}(o)$. Then $X_{t} \cong X_{t^{\prime}}$ for any $t, t^{\prime} \in Q_{o}$. Then by Schuster [26] $f_{Q_{0}}: X \times{ }_{T} Q_{o} \rightarrow Q_{o}$ is a trivial family. Hence the constant map $\tau: Q_{o} \rightarrow\{o\}$ 
is a versal map associated to $f_{Q_{0}}$ considered as a deformation of $X$. Since the differential $\tau_{*}$ of $\tau$ is zero and $\tau_{*}$ is unique, the inclusion $Q_{o} \rightarrow T$ must coincide with $\tau$, i.e., $Q_{o}=(o, o)$.

q.e.d.

In the above proof one can actually show that $r_{i}^{-1}(o) \cap Q=(o, o)$.

Proof of Proposition 7. Since $S$ is reduced, it suffices to show that $\operatorname{dim} H^{0}$ $\left(X_{s}, \Theta_{X_{s}}\right)$ is independent of $s$ in a neighborhood of $o$. This clearly follows from the same assertion for the Kuranishi family $(f: \mathscr{X} \rightarrow S, \widetilde{\omega})$ of $(X, \omega)=\left(X_{o}\right.$, $\left.\widetilde{\omega}_{o}\right)$ as above. So we shall prove the latter, using the above notations. For any $\left(t, t^{\prime}\right) \in Q, r_{J}^{-1}\left(t, t^{\prime}\right)$ is a union of connected components of $\operatorname{Isom}\left(X_{t}, X_{t^{\prime}}\right)$ $\neq \phi$ and hence $\operatorname{dim} r_{J}^{-1}\left(t, t^{\prime}\right)=\operatorname{dim}$ Aut $_{0} X_{t}=d(t):=\operatorname{dim} H^{0}\left(X_{t}, \Theta_{X_{t}}\right)$. Since $p_{1}$ : $=r_{1 \mid Q}$ is finite by Lemma 7 , there exist only a finite number of holomorphic sections, say $c_{1}, \cdots, c_{m}$, of $p_{1}$ defined on $T_{\text {red }}$. Let $Q_{i}=c_{i}\left(T_{\text {red }}\right) \subseteq Q$.

Suppose now that $d(t)$ is not constant on $T$. Then by the first remark the dimension of the general fiber of $r_{i}: r_{J}^{-1}\left(Q_{i}\right) \rightarrow Q_{i}$ is less than $d(o)$ on an irreducible component of $Q_{i}$. It follows that there exists a Zariski open subset $V$ of $J_{(o, o)}$ such that for any $v \in V$ and any $i$ there is no holomorphic section of $r_{i}$ passing through $v$. On the other hand, let $(\tilde{\nu}, \bar{v})$ be the automorphism of $(f, \widetilde{\omega})$ associated to $v \in J_{(o, o)}=\operatorname{Aut}_{0} X$ as in the proof of Lemma 6. Then $\tilde{\nu}$ is naturally regarded as a holomorphic section of $\gamma_{J}$ defined over the graph $\Gamma_{\bar{v}}$ $\subseteq T \times T$ of $\bar{v}$ and passing through $v$. Since $\Gamma_{\bar{v}}$ defines a holomorphic section of $p_{1}$, this is a contradiction. Hence $d(t)$ is constant. q.e.d.

Remark 5. The above proof actually works also for the Kuranishi family itself (not necessarily polarized). Indeed, the proof depends only on the fact that $\gamma_{J}$ is proper and the corresponding fact can be proved by a method similar to Proposition 2 using the $C^{\infty}$ extension theorem of Kähler metrics due to Kodaira-Spencer [18].

\section{§5. Local Modular Family}

In this section we shall construct the local modular family for a polarized compact Kähler manifold with $\operatorname{Aut}_{0} X$ a complex torus and give some of its basic properties.

(5.1) We start with the following:

Definition. Let $(f: \mathscr{X} \rightarrow S, \widetilde{\omega})$ be a polarized family of compact Kähler manifolds. Let $s \in S$. Then we say that $(f, \widetilde{\omega})$ is locally complete at $s$ if for 
any polarized deformation $\left(f^{\prime}: \mathscr{X}^{\prime} \rightarrow S^{\prime}, \widetilde{\omega}^{\prime}\right),\left(X_{s^{\prime}}^{\prime}, \widetilde{\omega}_{s^{\prime}}^{\prime}\right)=\left(X_{s}, \widetilde{\omega}_{s}\right), s^{\prime} \in S^{\prime}$, of $\left(X_{s}, \widetilde{\omega}_{s}\right)$ there exists a morphism $\tau: S^{\prime} \rightarrow S, \tau\left(s^{\prime}\right)=S$, such that $\left(f^{\prime}, \widetilde{\omega}^{\prime}\right)$ is isomorphic to the pull-back $\left(f_{S^{\prime}}, \widetilde{\omega}_{S^{\prime}}\right)$ of $(f, \widetilde{\omega})$ to $S^{\prime}$. If, further, $\tau$ is unique for any $\left(f^{\prime}, \widetilde{\omega}^{\prime}\right)$ as above, we say that $(f, \widetilde{\omega})$, considered as a polarized deformation of $\left(X_{s}, \widetilde{\omega}_{s}\right)$, is the local modular family of $\left(X_{s}, \widetilde{\omega}_{s}\right)$. In this case $\tau$ is called the universal map associated to $\left(f^{\prime}, \widetilde{\omega}^{\prime}\right)$.

Proposition 8. For any polarized compact Kähler manifold $(X, \omega)$ such that $\mathrm{Aut}_{0} X$ is a complex torus its local modular family $(f: \mathcal{X} \rightarrow S, \widetilde{\omega}),\left(X_{o}, \widetilde{\omega}_{o}\right)$ $=(X, \omega), o \in S$, exists. Moreover it is locally complete at every point in a neighborhood of $o$.

Proof. Let $f_{1}: \mathscr{X}_{1} \rightarrow S_{1}, X_{1,0}=X, o \in S_{1}$, be the Kuranishi family of $X$ [19]. Let $\widetilde{\omega}_{1} \in \Gamma\left(S_{1}, R^{2} f_{1} \mathbb{R}\right)$ be the element corresponding to $\omega$ with respect to the isomorphism (*) in (3.1) for $f_{1}$. Let $S$ be the subspace of $S_{1}$ defined by the equation $\eta\left(\widetilde{\omega}_{1}\right)=0$, where $\eta: \Gamma\left(S_{1}, R^{2} f_{1^{*}} \mathbb{R}\right) \rightarrow \Gamma\left(S_{1}, R^{2} f_{1^{*}} \mathcal{O}_{\mathfrak{X}_{1}}\right)$ is the natural homomorphism. (Note that since $R^{2} f_{1^{*}} \mathcal{O}_{\mathfrak{X}_{1}} \cong \mathcal{O}_{S_{1}}$ for some $r \geqq 0$ by Proposition 1, the equation is just a system of $r$ ordinary equations.) Let $f: \mathscr{X} \rightarrow S$ and $\tilde{\omega} \in \Gamma\left(S, R^{2} f_{*} \mathbb{R}\right)$ be the restriction of $f_{1}$ and $\widetilde{\omega}_{1}$ to $S$ respectively. Then we claim that $(f, \widetilde{\omega})$ thus obtained is 1$)$ a polarized deformation of $(X, \omega)$, 2) locally complete at any point of $s$ in a neighborhood of $o$, and finally, 3) the local modular family of $(X, \omega)$.

1) By our construction $(f, \widetilde{\omega})$ clearly satisfies the condition (2) of Definition in (1.1). Thus it suffices to show that $\widetilde{\omega}_{s}$ is a Kähler class on $X_{s}$ for any $s \in S$ in a neighborhood of $o$. Let $\pi: \hat{S} \rightarrow S_{\text {red }}$ be a resolution of $S_{\text {red. }}$ Let $(\hat{f}: \hat{X} \rightarrow \hat{S}, \hat{\omega})$ be the pull-back of $(f, \widetilde{\omega})$ to $\hat{S}$. Then $(\hat{f}, \hat{\omega})$ satisfies the condition 1) of Lemma 1 at each point of $\pi^{-1}(o)$. Hence by that lemma $\hat{\omega}_{\hat{s}}$ is a Kähler class on $X_{\hat{s}}$ for any $\hat{s}$ in a neighborhood of $\pi^{-1}(o)$. It follows that $\widetilde{\omega}_{s}$ is a Kähler class for any $s$ in a neighborhood of $o$.

2) Let $\left(f^{\prime}: \mathscr{X}^{\prime} \rightarrow S^{\prime}, \widetilde{\omega}^{\prime}\right),\left(X_{s^{\prime}}^{\prime}, \widetilde{\omega}_{s^{\prime}}\right)=\left(X_{s}, \widetilde{\omega}_{s}\right), s^{\prime} \in S^{\prime}$, be any polarized deformation of $\left(X_{s}, \widetilde{\omega}_{s}\right)$. By virtue of the local completeness of the original Kuranishi family $f_{1}$ (if $s$ is sufficiently near to $o$ ) we get a versal map $\tau: S^{\prime} \rightarrow S$ induced by $f^{\prime}: \mathscr{X}^{\prime} \rightarrow S^{\prime}$ considered as a deformation of $X_{s^{\prime}}^{\prime}=X_{s}$ with the differential $\tau_{*}$ unique when $s=o$. Then since $\eta_{f^{\prime}}\left(\widetilde{\omega}^{\prime}\right)=0$, by (2.3) we conclude that $\tau$ actually factors through $S \subseteq S_{1}$. It is then immediate to see that the pullback $\left(f_{S^{\prime}}, \widetilde{\omega}_{S^{\prime}}\right)$ of $(f, \widetilde{\omega})$ to $S^{\prime}$ is isomorphic to $\left(f^{\prime}, \widetilde{\omega}^{\prime}\right)$.

3) Finally in case $s=o$ the uniqueness of $\tau$ above follows from the cohomological flatness of $\Theta \mathscr{X} / S$ shown in Theorem 1 by (completely the same 
argument as) Wavrik [29] or Palamodov [25].

q.e.d.

Remark 6. In the general case (i.e., the case where Aut $_{0} X$ is not necessarily a complex torus) $(f, \widetilde{\omega})$ defined above is not necessarily the local modular family (cf. [29] [25]). However, in view of the properties 1) and 2) proved above we shall call it the (polarized) Kuranishi family of $(X, \omega)$.

Remark 7. The Zariski tangent space of the parameter space $S$ at $o$ is naturally identified with the kernel $H^{1}\left(X, \Theta_{X}\right)_{\omega}$ of the linear map $H^{1}\left(X, \Theta_{X}\right) \rightarrow$ $H^{2}\left(X, \Theta_{X}\right)$ defined by the cup product with $\omega$, considered as an element of $H^{1}\left(X, \Omega_{X}^{1}\right)$.

(5.2) Let $(X, \omega)$ be a polarized compact Kähler manifold such that $\mathrm{Aut}_{0} X$ is a complex torus. Let $(f: \mathfrak{X} \rightarrow S, \widetilde{\omega}),\left(X_{o}, \widetilde{\omega}_{o}\right)=(X, \omega), o \in S$, be the local modular family of $(X, \omega)$. Then the next lemma shows the topological significance of considering local modular family in the study of the local structure of $\mathfrak{M}$.

Lemma 8. Take $S$ sufficiently small so that $(f, \widetilde{\omega})$ is locally complete at any point of $S$. Then the canonical map $\rho=\rho(f, \widetilde{\omega}):|S| \rightarrow \mathfrak{M}$ is an open map so that the image $\bar{S}:=\rho(|S|)$ is an open neighborhood of $(X, \omega)$ in $\mathfrak{M e}$.

Proof. Let $U \subseteq S$ be any open subset. It suffices to show that for any polarized family of compact Kähler manifolds $\left(f^{\prime}: \mathfrak{X}^{\prime} \rightarrow S^{\prime}, \widetilde{\omega}^{\prime}\right), U^{\prime}:=\rho^{\prime-1}(\rho(U))$ is open in $S^{\prime}$, where $\rho^{\prime}=\rho\left(f^{\prime}, \widetilde{\omega}^{\prime}\right)$. Let $s^{\prime} \in U^{\prime}$ be an arbitrary point. Take a point $s \in U$ with $\rho^{\prime}\left(s^{\prime}\right)=\rho(s):=m_{1}$. Let $\left(X_{1}, \omega_{1}\right)$ be the polarized compact Kähler manifold corresponding to $m_{1}$. Let $\left(f_{1}: \mathscr{X}_{1} \rightarrow S_{1}, \widetilde{\omega}_{1}\right),\left(X_{1, o_{1}}, \widetilde{\omega}_{1, o_{1}}\right)=$ $\left(X_{1}, \omega_{1}\right), o_{1} \in S_{1}$, be the local modular family of $\left(X_{1}, \omega_{1}\right)$. (We may assume that Aut $X_{1}$ is also a complex torus.) Let $\tau: V \rightarrow S_{1}, \tau(s)=o_{1}$ (resp. $\tau^{\prime}: V^{\prime} \rightarrow S_{1}$, $\left.\tau^{\prime}\left(s^{\prime}\right)=o_{1}\right)$ be the corresponding universal map, where $V$ (resp. $V^{\prime}$ ) is a small neighborhood of $s$ (resp. $s^{\prime}$ ).

On the other hand, by the local completeness of $(f, \widetilde{\omega})$ at $s$ we have a versal map $\tau_{1}: V_{1} \rightarrow S$ with $\tau_{1}\left(o_{1}\right)=S$, where $V_{1}$ is a sufficiently small neighborhood of $o_{1}$ in $S_{1}$. Then by the modularity of $\left(f_{1}, \widetilde{\omega}_{1}\right)$ at $o_{1}, \tau \tau_{1}$ must be the identity of $V_{1}$. Hence if we take $V^{\prime}$ sufficiently small, $\tau^{\prime}\left(V^{\prime}\right) \subseteq V_{1} \subseteq \tau(V)$. Now let $\rho_{1}=\rho\left(f_{1}, \widetilde{\omega}_{1}\right)$. Then we have $\rho_{1} \tau=\rho_{\mid V}$ and $\rho_{1} \tau^{\prime}=\rho^{\prime}{ }_{\mid V^{\prime}}$. Hence $\rho^{\prime}\left(V^{\prime}\right) \subseteq \rho_{1} \tau(V)$ $=\rho(V)$, and so $V^{\prime} \subseteq \rho^{\prime-1}(\rho(U))=U^{\prime}$. Since $s^{\prime}$ was arbitrary, $U^{\prime}$ is open. q.e.d.

(5.3) Let $(X, \omega)$ be a polarized compact Kähler manifold such that Aut $_{0} X$ is a complex torus. Let $(f: \mathscr{X} \rightarrow S, \widetilde{\omega}),\left(X_{o}, \widetilde{\omega}_{o}\right)=(X, \omega), o \in S$, be the local 
modular family of $(X, \omega)$. Let $\left(f^{\prime}: \mathfrak{X}^{\prime} \rightarrow S^{\prime}, \widetilde{\omega}^{\prime}\right)$ be any polarized deformation of $\left(X_{o^{\prime}}^{\prime}, \widetilde{\omega}_{o^{\prime}}^{\prime}\right), o^{\prime} \in S^{\prime}$, with an isomorphism $b:\left(X_{o^{\prime}}^{\prime}, \widetilde{\omega}_{o^{\prime}}^{\prime}\right) \underset{\rightarrow}{\rightarrow}(X, \omega)$. Then there exists a morphism $(\tilde{\tau}, \tau):\left(f^{\prime}, \widetilde{\omega}^{\prime}\right) \rightarrow(f, \widetilde{\omega})$ of polarized deformations, i.e., morphisms $\tilde{\tau}: \mathscr{X}^{\prime} \rightarrow \mathfrak{X}$ and $\tau: S^{\prime} \rightarrow S$ with $\tau f^{\prime}=f \tilde{\tau}$ and $\tau\left(o^{\prime}\right)=o$, such that $\tilde{\tau}_{o^{\prime}}=b$, where $\tilde{\tau}_{o^{\prime}}:\left(X_{o^{\prime}}^{\prime}, \widetilde{\omega}_{o^{\prime}}^{\prime}\right) \rightarrow\left(X, \widetilde{\omega}_{o}\right)=(X, \omega)$ is the isomorphism induced by $\tilde{\tau}$. Moreover $\tau$ is unique by the modularity of $(f, \widetilde{\omega})$. We call this $\tau$ the universal map associated with $\left(f^{\prime}, \widetilde{\omega}^{\prime}\right)$ with respect to the isomorphism $b$.

When $\left(f^{\prime}, \widetilde{\omega}^{\prime}\right)=(f, \widetilde{\omega})$ (so that $\mathscr{X}=\mathscr{X}^{\prime}$ and $\left.S^{\prime}=S\right), b$ is an automorphism of $(X, \omega)$ and the resulting $\tilde{\tau}$ and $\tau$ are automorphisms of $\mathscr{X}$ and $S$ respectively (cf. the proof of Lemma 6). Thus letting $\delta_{0}(b)=\tau$ we get a map $\delta_{0}: \operatorname{Aut}(X, \omega)$ $\rightarrow$ Aut $S(=$ Aut $(S, o))$, which turns out to be a homomorphism.

Lemma 9. Aut $_{0} X$ is contained in the kernel of $\delta_{0}$.

Proof. Let $b \in \mathrm{Aut}_{0} X$ be arbitrary. Since Aut $\mathscr{X} / S$ is smooth along Aut $_{0} X \cong(\text { Aut } \mathscr{X} / S)_{o}$ by Proposition 6 , there exists a holomorphic section $\mu$ : $S \rightarrow$ Aut $\mathscr{X} / S$ with $\mu(o)=b$. This defines an automorphism $\tilde{\tau}$ of $\mathscr{X}$ over $S$. By the definition of $\delta_{0}$, this implies that $\delta_{0}(b)$ is the identity of $S$. q.e.d.

Set $H=H(X, \omega):=\operatorname{Aut}(X, \omega) / \operatorname{Aut}_{0} X$. Then $H$ is a finite group by Corollary to Proposition 2. By Lemma $9 \delta_{0}$ induces a homomorphism $\delta: H \rightarrow$ Aut $S$. By the definition of $\delta$ for any $h \in H$ there exists an automorphism $(\tilde{h}, \bar{h})$ of $(f, \widetilde{\omega})$;

$$
\begin{gathered}
\mathscr{X} \stackrel{\tilde{h}}{\longrightarrow} \mathscr{X} \\
f \downarrow \\
S \stackrel{\bar{h}}{\longrightarrow} S f \\
S,
\end{gathered}
$$

such that $q\left(\tilde{h}_{o}\right)=h$, where $\tilde{h}_{o} \in \operatorname{Aut}(X, \omega)$ is the automorphism induced by $\tilde{h}$, $q$ : $\operatorname{Aut}(X, \omega) \rightarrow H(X, \omega)$ is the quotient homomorphism and where $\bar{h}=\delta(h)$.

On the other hand, the quotient space $S / H$ admits a natural complex space structure so that the quotient map $\pi: S \rightarrow S / H$ is a morphism of complex spaces. Then the canonical map $\rho(f, \widetilde{\omega}):|S| \rightarrow \bar{S}$ obviously factors as

$$
|S| \stackrel{|\pi|}{\longrightarrow}|S / H| \stackrel{\bar{\rho}}{\rightarrow} \bar{S} \text {. }
$$

In the next section we shall show that $\bar{\rho}$ is homeomorphic under a suitable assumption on $(X, \omega)$.

\section{§6. Definition and the Analytic Structure of $\mathfrak{B}$}

(6.1) Let $(X, \omega)$ be a polarized compact Kähler manifold such that $\mathrm{Aut}_{0} X$ 
is a complex torus. Let $(f: \mathscr{X} \rightarrow S, \widetilde{\omega}),\left(X_{o}, \widetilde{\omega}_{o}\right)=(X, \omega), o \in S$, be the local modular family of $(X, \omega)$. Let $p_{i}: S \times S \rightarrow S, i=1,2$, be the projection to the $i$-th factor. Let $\left(f_{i}: \mathfrak{X}_{i} \rightarrow S \times S, \widetilde{\omega}_{i}\right)$ be the pull-back of $(f, \widetilde{\omega})$ via $p_{i}$. Set $I$ $=\operatorname{Isom}_{S \times S}\left(\left(\mathfrak{X}_{1}, \widetilde{\omega}_{1}\right),\left(\mathfrak{X}_{2}, \widetilde{\omega}_{2}\right)\right)$. Let $r: I \rightarrow S \times S$ be the natural morphism. $r$ is up to isomorphism uniquely determined by $(X, \omega)$ as a germ over $(o, o) \in S \times S$. By the definition we have the natural identification $I_{(o, 0)}=\operatorname{Aut}(X, \omega)$. Using $I$ we shall define the subset $\mathfrak{B}$ of $\mathfrak{M}$ as follows.

Definition. $\mathfrak{B}=\{(X, \omega) \in \mathfrak{M} ; r$ is proper over $S \times S$ in a neighborhood of $(o, o)\}$.

In particular if $(X, \omega) \in \mathfrak{B}, \operatorname{Aut}(X, \omega)$ is compact and $\mathrm{Aut}_{0} X$ is a complex torus. Further if Aut $_{0} \mathfrak{X} / S$ is the connected component of Aut $\mathscr{X} / S$ containing Aut $_{0} X \subseteq(\text { Aut } \mathscr{X} / S)_{o}$, Aut ${ }_{0} \mathscr{X} / S$ is proper, smooth and with connected fibers over $S$ (cf. Theorem 1 and Proposition 6). We also remark that by Proposition 4 if $(X, \omega) \in \mathfrak{M}-\mathfrak{B}$, then $X$ is ruled.

Now the purpose of this section is to prove the following:

Theorem 2. The coarse $\mathfrak{B}$-moduli space $(B, \psi)$ exists except that $B$ is not separated.

(6.2) In this and the next subsections we shall fix a polarized compact Kähler manifold $(X, \omega)$ in $\mathfrak{B}$ and its local modular family $(f: \mathscr{X} \rightarrow S, \widetilde{\omega}),\left(X_{o}, \widetilde{\omega}_{o}\right)=$ $(X, \omega), o \in S$. We shall show that $\bar{\rho}:|S / H| \rightarrow \bar{S}$ defined in (5.3) is homeomorphic. We set

$$
R=\left\{\left(s_{1}, s_{2}\right) \in|S| \times|S| ;\left(X_{s_{1}}, \widetilde{\omega}_{s_{1}}\right) \cong\left(X_{s_{2}}, \widetilde{\varpi}_{s_{2}}\right)\right\} .
$$

Then $R$ defines an equivalence relation on $|S|$, and the quotient $|S| / R$ of $|S|$ with respect to this equivalence relation endowed with the quotient topology is naturally homeomorphic to $\bar{S}$ (cf. Lemma 8). Let $H=H(X, \omega)$ be as in (5.3). Then the set of connected components of $\operatorname{Aut}(X, \omega)$ is canonically indexed by $H$. Namely $\operatorname{Aut}(X, \omega)=\prod_{h \in H} A^{h}$ with $A^{h}=q^{-1}(h)$, where $q: \operatorname{Aut}(X, \omega)$ $\rightarrow H(X, \omega)$ is the quotient homomorphism. Let $I^{h}$ be the connected component of $I$ containing $A^{h}$ with respect to the natural identification $I_{(o, o)}=\operatorname{Aut}(X$, $\omega)$. Then $I=\|_{h \in B} I^{h}$. (Recall that we are considering $I$ as a germ over $(o, o)$.)

Lemma 10. For any $h \in H, r\left(I^{h}\right)=R^{h}$, where $R^{h} \subseteq S \times S$ is the graph of $\bar{h}:=\delta(h) \in$ Aut $S$. Moreover the induced morphism $\gamma^{h}: I^{h} \rightarrow R^{h}$ is proper, smooth and with connected fibers. In particular $R=\cup_{h}\left|R^{h}\right|$. 
Proof. Let $\left(\hat{f}_{i}, \hat{\omega}_{i}\right)$ be the pull-back of $\left(f_{i}, \widetilde{\omega}_{i}\right)$ to $I^{h}$ via $r l_{I^{k}}$. We have the canonical $I^{h}$-isomorphism $\lambda:\left(\hat{\mathcal{X}}_{1}, \hat{\omega}_{1}\right) \rightarrow\left(\hat{\mathfrak{X}}_{2}, \hat{\omega}_{2}\right)$. On the other hand, for any $a \in I_{(o, o)}^{h}$ we have the canonical identification $\left(\hat{X}_{2, a}, \hat{\omega}_{2, a}\right)=(X, \omega)$. In particular $\lambda$ induces an isomorphism $\tilde{\lambda}_{a}:\left(\hat{X}_{1, a}, \hat{\omega}_{1, a}\right) \cong(X, \omega)$. Let $\tau:\left(I^{h}, a\right)$ $\rightarrow(S, o)$ be the universal map associated with $\left(\hat{f}_{1}, \hat{\omega}_{1}\right)$ with respect to $\tilde{\lambda}_{a}$ (cf. (5.3)). Then we may write $\tau=\bar{h} p_{1} \gamma$ and also $\tau=p_{2} r$; indeed as the corresponding morphism $\tilde{\tau}: \hat{\mathfrak{X}}_{1} \rightarrow \mathfrak{X}$ which covers $\tau$ as in (5.3) we can take, in the first case, the composition of the natural maps $\hat{\mathscr{X}}_{1} \rightarrow \mathfrak{X}_{1} \rightarrow \mathfrak{X} \rightarrow \mathfrak{W}$ where $\tilde{h}$ is as in $(\dagger)$, and in the second case, $\lambda$ followed by the natural projection $\hat{\mathscr{X}}_{2} \rightarrow \mathscr{X}_{2} \rightarrow \mathscr{X}$. Thus $\bar{h} p_{1} r=p_{2} r$. Since $a$ was arbitrary, this implies that $r\left(I^{h}\right) \subseteq R^{h}$.

For the other inclusion, we have only to show the existence of a holomorphic section $b: R^{h} \rightarrow I^{h}$. Let $(\tilde{h}, \bar{h})$ be as above, i.e., any automorphism of $(f, \widetilde{\omega})$ defined by any element of $A^{h}(\mathrm{cf} .(\dagger))$. Then $\tilde{h}$ induces an isomorphism $\hat{h}$ of $\left(f_{1}, \widetilde{\omega}_{1}\right)$ and $\left(f_{2}, \widetilde{\omega}_{2}\right)$ over $R^{h}$ with $\hat{h}_{(o, o)} \in A^{h}$, which in turn gives a desired holomorphic section $b$. Further the existence of such a section implies that $I^{h}$ is a relative principal homogeneous space over $R^{h}$ with respect to the relative

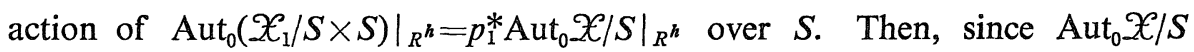
is smooth and proper over $S$ with connected fibers, and hence so is $p_{1}^{*}\left(\right.$ Aut $_{0}$ $\mathscr{X} / S)\left.\right|_{R^{h}}$ over $R^{h}$, the same is true for $I^{h} \rightarrow R^{h}$. Finally, by what we have proved above, the last equality follows from the relation $R=r(I)$.

q.e.d.

From the last relation we get

Corollary. $\bar{\rho}:|S / H| \rightarrow \bar{S}$ is homeomorphic.

In view of Lemma 10, in what follows we consider $R$ as a complex subspace (not necessarily reduced) of $S \times S$ by the equality $R=\cup_{h} R^{h}$. Then $r$ induces a morphism $I \rightarrow R$ of complex space (which will still be denoted by $r$ ). Then $r$ is factored as

$$
I=\Perp_{h} I^{h} \rightarrow \widetilde{R}:=\Perp_{h} R^{h} \stackrel{\bar{r}}{\rightarrow} R
$$

where the first arrow is given by $\Perp_{h} \gamma^{h}$ and $\left.\bar{\gamma}\right|_{R^{h}}$ is the natural inclusion $R^{h} \rightarrow$ $R$. Note that $p_{i} \bar{\gamma}: \widetilde{R} \rightarrow S$ is a finite unramified covering.

(6.3) We can now define a complex space structure on $\bar{S}$ (not necessarily reduced) by transplanting that of $S / H$ via the homeomorphism $\bar{\rho}:|S / H| \rightarrow \bar{S}$. We want to see that at any $\left(X^{\prime}, \omega^{\prime}\right) \in \bar{S}$ this complex structure on $\bar{S}$ is canonically isomorphic to the one which is defined as above starting from the local modular family of $\left(X^{\prime}, \omega^{\prime}\right)$. For this purpose we first note the following fact 
which is easily seen from our construction. Let $s \in S$. Let $H(X, \omega)_{s}=\{h \in$ $H(X, \omega) ; \bar{h} s=s\}$. Then there exists a natural isomorphism $\psi_{s}: H(X, \omega)_{s} \sim$ $H\left(X_{s}, \widetilde{\omega}_{s}\right)$ defined by $\psi_{s}(h)=q_{s}\left(I_{(s, s)}^{h}\right), h \in H(X, \omega)_{s}$, with respect to the natural identification $I_{(s, s)}=\operatorname{Aut}\left(X_{s}, \widetilde{\omega}_{s}\right)$, where $q_{s}: \operatorname{Aut}\left(X_{s}, \widetilde{\omega}_{s}\right) \rightarrow H\left(X_{s}, \widetilde{\omega}_{s}\right)=\operatorname{Aut}\left(X_{s}\right.$, $\left.\widetilde{\omega}_{s}\right) /$ Aut $_{0} X_{s}$ is the natural homomorphism.

In view of this remark our remaining task is to show the next proposition, which is indeed a special case of a theorem of Palamodov (cf. $\left[26,7^{\circ}\right]$ ). Since his proof is rather involved (using his own construction of the Kuranishi family), we shall give a simpler proof in our special case.

Proposition 9. For any $s \in S$ which is sufficiently near to $o \in S,(f, \widetilde{\omega})$ gives the local modular family for $\left(X_{s}, \widetilde{\omega}_{s}\right)$ also.

Proof. Take $S$ small so that $(f, \widetilde{\omega})$ is locally complete at any point of $S$ and that $\left(X_{s}, \widetilde{\omega}_{s}\right) \in \mathfrak{B}$ for any $s \in S$. Fix any $s \in S$. Let $\left(f^{\prime}: \mathscr{X}^{\prime} \rightarrow S^{\prime}, \widetilde{\omega}^{\prime}\right),\left(X_{o}^{\prime}\right.$, $\left.\widetilde{\omega}_{o^{\prime}}^{\prime}\right)=\left(X_{s}, \widetilde{\omega}_{s}\right), o^{\prime} \in S^{\prime}$, be the local modular family for $\left(X_{s}, \widetilde{\omega}_{s}\right)$. Let $\tau:(S$, $s) \rightarrow\left(S^{\prime}, o^{\prime}\right)$ be the universal map obtained by considering $(f, \tilde{\omega})$ as a deformation of $\left(X_{s}, \widetilde{\omega}_{s}\right)$. Let $\tau^{\prime}:\left(S^{\prime}, o^{\prime}\right) \rightarrow(S, s)$ be a versal map induced by the local completeness of $(f, \widetilde{\omega})$ at $s$. By the modularity of $\left(f^{\prime}, \widetilde{\omega}^{\prime}\right), \tau \tau^{\prime}$ is the identity of $S^{\prime}$ at $o^{\prime}$. In particular $\tau$ is surjective.

Thus it suffices to show that $\tau$ is an embedding, which in turn would follow if we show that the natural inclusion $\Delta_{S} \subseteq(\tau \times \tau)^{-1}\left(\Delta_{S^{\prime}}\right)$ is an equality where for $Y=S, S^{\prime}, \Delta_{Y}$ is the diagonal of $Y \times Y$.

Let $I$ and $I^{e}$ be as in (6.2) (for $(f, \widetilde{\omega})$ ), where $e$ is the identity of $H=H(X$, $\omega)$. Then by Lemma $10 I^{e}$ is smooth over $\Delta_{S}$ with $I_{(s, s)}^{e}=$ Aut $_{0} X_{s}$ with respect to the natural identification $I_{(s, s)}=\operatorname{Aut}\left(X_{s}, \widetilde{\omega}_{s}\right)$. Let $\left(f_{i}^{\prime}: \mathscr{X}_{i}^{\prime} \rightarrow S^{\prime} \times S^{\prime}, \widetilde{\omega}_{i}^{\prime}\right)$ be the pull-back of $\left(f^{\prime}, \widetilde{\omega}^{\prime}\right)$ to $S^{\prime} \times S^{\prime}$ via $p_{i}: S^{\prime} \times S^{\prime} \rightarrow S^{\prime}$, the projection to the $i$-th factor. Let

$$
' I=\operatorname{Isom}_{S^{\prime} \times S^{\prime}}\left(\left(\mathscr{X}_{1}^{\prime}, \widetilde{\omega}_{1}^{\prime}\right),\left(\mathscr{X}_{2}^{\prime}, \widetilde{\omega}_{2}^{\prime}\right)\right)
$$

We then get the natural decomposition ' $I=\Perp^{\prime} I^{h}, h \in H\left(X_{s}, \widetilde{\omega}_{s}\right)$ as in (6.2). Further ' $I^{e}$ is the unique connected component of ' $I^{e}$ with ' $I^{e}=\mathrm{Aut}_{0} X_{s}$, where we denote the unit element of $H\left(X_{s}, \widetilde{\omega}_{s}\right)$ again by $e$. Let ' $\tilde{I}=' I \times{ }_{\left(S^{\prime} \times S^{\prime}\right)}(S \times S)$ and ' $\tilde{I}^{e}={ }^{\prime} I^{e} \times{ }_{\left(S^{\prime} \times S^{\prime}\right)}(S \times S)$, where $S \times S$ is over $S^{\prime} \times S^{\prime}$ by $\tau \times \tau$. Then ' $\tilde{I}^{e}$ is smooth over $(\tau \times \tau)^{-1}\left(\Delta_{S^{\prime}}\right)$. Since we must have a natural isomorphism $I \cong{ }^{\prime} \tilde{I}$ which induces the identity $I_{(s, s)}={ }^{\prime} \widetilde{I}_{(s, s)}\left(=\operatorname{Aut}\left(X_{s}, \widetilde{\omega}_{s}\right)\right.$ ) we get $I^{e}={ }^{\prime} \widetilde{I}^{e}$ over $S \times S$. Thus ' $\tilde{I}^{e}$ is smooth also over $\Delta_{S}$. Hence $(\tau \times \tau)^{-1}\left(\Delta_{S^{\prime}}\right)=\Delta_{S}$ as was desired. 
In what follows when we speak of the local modular family $(f: \mathfrak{X} \rightarrow S, \widetilde{\omega})$ we always assume that the condition of the above proposition is satisfied for any $s \in S$.

(6.4) Proof of Theorem 2. i) Existence of complex structure. By Lemma 8 and by Proposition 9 we see that $\mathfrak{B}$ is open in $\mathfrak{M}$. Let $(X, \omega)$ be any point of $\mathfrak{B}$. Let $(f: \mathscr{X} \rightarrow S, \widetilde{\omega})$ be the local modular family of $(X, \omega)$. By Lemma 8 $\bar{S}=\rho(f, \widetilde{\omega})(|S|)$ is an open neighborhood of $(X, \omega)$ in $\mathfrak{B}$, and by Corollary to Lemma $10 \rho(f, \widetilde{\omega})$ induces a homeomorphism $\bar{\rho}:|S / H| \rightarrow \bar{S}$. Then we define a complex space structure on $\bar{S}$ by transplanting the natural one on $S / H$ via $\bar{\rho}$ so that $\rho(f, \widetilde{\omega})$ is induced from a morphism of complex spaces $S \rightarrow \bar{S}$, which we shall still denote by $\rho(f, \widetilde{\omega})$. By Proposition 9 together with a remark just before that proposition it follows that for each $\left(X^{\prime}, \omega^{\prime}\right) \in \bar{S}$ the complex space structure of $\bar{S}$ in a neighborhood of $\left(X^{\prime}, \omega^{\prime}\right)$ by the above procedure is canonically isomorphic to the complex space structure on $\bar{S}$ just obtained. Since $\mathfrak{B}$ is covered by open subsets of the form $\rho(f, \widetilde{\omega})(|S|)$ with varying $(X, \omega)$ and $(f, \widetilde{\omega})$, it follows readily that we get a global complex space structure on $\mathfrak{B}$ in this way. Let $B$ be the resulting complex space (not necessarily separated).

ii) Existence of the functorial morphism $\mathscr{B} \rightarrow h_{B}$. Let $(g: \mathscr{\jmath} \rightarrow T, \widetilde{\omega})$ be any polarized $\mathfrak{B}$-family of compact Kähler manifolds. Then for any $t \in T$ there exist a neighborhood $t \in U_{t}$ and the universal map $\tau_{t}: U_{t} \rightarrow S^{t}$, where $\left(f^{t}: \mathscr{X}^{t} \rightarrow S^{t}, \widetilde{\omega}^{t}\right)$ is the local modular family of $\left(Y_{t}, \widetilde{\omega}_{t}\right)$. Composing this with $\rho\left(f^{t}, \widetilde{\omega}^{t}\right): S^{t} \rightarrow \bar{S}^{t}=S^{t} / H\left(Y_{t}, \widetilde{\omega}_{t}\right) \subseteq B$ we have a morphism $\bar{\tau}_{t}: U_{t} \rightarrow B$. For any $t^{\prime} \in U_{t}$, if we take a neighborhood $U_{t^{\prime}}$ of $t^{\prime}$ in $T$ and obtain a morphism $\bar{\tau}_{t^{\prime}}$ : $U_{t^{\prime}} \rightarrow B$ by the same procedure as above, then from Proposition 9 it follows that $\bar{\tau}_{t}$ and $\bar{\tau}_{t^{\prime}}$ coincide at $t^{\prime}$. Since $T$ is covered by $U_{t}$ as above with $t$ varying on $T$, from this we see readily that $\bar{\tau}_{t}, t \in T$, patch together to give a global morphism $\bar{\tau}: T \rightarrow B$. Then the correspondence $(g, \widetilde{\omega}) \rightarrow \bar{\tau}$ defines a desired morphism of functors $\psi: \mathscr{B} \rightarrow h_{B}:=\operatorname{Hom}(, B)$.

iii) We show that the pair $(B, \psi)$ is the desired coarse $\mathfrak{B}$-moduli space. Let $B_{1}$ be any complex space with a functorial morphism $\psi_{1}: \mathscr{B} \rightarrow h_{B_{1}}$ Take any $(X, \omega) \in \mathfrak{B}$. Let $(f: \mathscr{X} \rightarrow S, \widetilde{\omega})$ be the local modular family of $(X, \omega)$. Then $\psi_{1}$ defines a unique morphism $\tau_{1}: S \rightarrow B_{1}$ associated to $(f, \tilde{\omega})$. We shall show that $\tau_{1}$ factors through $\rho(f, \widetilde{\omega}): S \rightarrow \bar{S}$. Take any automorphism $(\tilde{h}, \bar{h})$ of $(f, \widetilde{\omega})$ as in $\left(^{\dagger}\right)$. This then defines an isomorphism over $S$ of $(f, \widetilde{\omega})$ and the pull-back $\bar{h}^{*}(f, \widetilde{\omega})$ of $(f, \widetilde{\omega})$ by $\bar{h}$. Since the universal map associated to $\bar{h}^{*}(f, \widetilde{\omega})$ is $\tau_{1} \bar{h}$, we must have $\tau_{1} \bar{h}=\tau_{1}$. Our assertion thus follows, $(\tilde{h}, \bar{h})$ being arbitrary. Let $\bar{\tau}_{1}=\bar{\tau}_{1}(X, \omega): \bar{S} \rightarrow B_{1}$ be the induced morphism. Then by the same argu- 
ment as in ii) all the $\bar{\tau}_{1}(X, \omega)$ defined for varying $(X, \omega)$ and $(f, \tilde{\omega})$ as above patch together to define a global morphism $\bar{\tau}_{1}: B \rightarrow B_{1}$. By our construction of $\psi$ we conclude easily that $\psi_{1}=\operatorname{Hom}\left(, \bar{\tau}_{1}\right) \psi$.

q.e.d.

\section{§7. Proof of Theorem}

(7.1) First we consider the problem of separation.

Proposition 10. Let $\mathfrak{R}=\{(X, \omega) \in \mathfrak{M} ; X$ is ruled $\}$. Then $\mathfrak{M}-\mathfrak{R}$ is separated with respect to the canonical topology of $\mathfrak{M}$.

Proof. Let $\Delta \subseteq(\mathfrak{M}-\mathfrak{R}) \times(\mathfrak{M}-\mathfrak{R})$ be the diagonal. Let $\left(m_{1}, m_{2}\right) \in \Delta^{-}$, where $\Delta^{-}$is the closure of $\Delta$. Take a sequence $\left(m_{1 k}, m_{2 k}\right), k=1,2, \cdots$, of points of $\Delta$ (so that $\left.m_{1 k}=m_{2 k}\right)$, converging to $\left(m_{1}, m_{2}\right)$. Let $m_{i}=\left(X_{i}, \omega_{i}\right)$. Let $\left(f_{i}\right.$ : $\left.\mathscr{X}_{i} \rightarrow S_{i}, \widetilde{\omega}_{i}\right)$ be the local modular family of $\left(X_{i}, \omega_{i}\right)$ with the base point $s_{i 0} \in S_{i}$. Then by Lemma 8 there exists a sequence $\left\{s_{i k}\right\}$ of points of $S_{i}$ converging to $s_{i 0}$ and with $\rho_{i}\left(s_{i k}\right)=m_{i k}$. Let $S=S_{1} \times S_{2}$ and $p_{i}: S \rightarrow S_{i}$ be the natural projection. Let $\left(\hat{f}_{i}: \hat{\mathcal{X}}_{i} \rightarrow S, \hat{\omega}_{i}\right)$ be the pull-back of $\left(f_{i}, \widetilde{\omega}_{i}\right)$ to $S$ by $p_{i}$. Then the set $S_{1}=\left\{\left(s_{1}, s_{2}\right) \in S ;\left(X_{1 s_{1}}, \widetilde{\omega}_{1 s_{1}}\right) \cong\left(X_{2 s_{2}}, \widetilde{\omega}_{2 s_{2}}\right)\right\}$ is a closed analytic subset of $S$ by Corollary to Proposition 4. Hence $\left(s_{10}, s_{20}\right) \in S_{1}$ since $\left(s_{1 k}, s_{2 k}\right) \in S_{1}$. Namely $m_{1}=\rho_{1}\left(s_{10}\right)=\rho_{2}\left(s_{20}\right)=m_{2}$. Thus $\Delta^{-}=\Delta$ since $\mathfrak{M} \times \mathfrak{M}$ satisfies the first countability axiom as follows from Lemma 8 .

q.e.d.

The following is a refinement of the main result of [8].

Proposition 11. Let $(f: \mathscr{X} \rightarrow S, \widetilde{\omega})$ be a polarized family of compact Kähler manifolds with $S$ connected. If $X_{o}$ is uniruled for some $o \in S$, then $X_{s}$ is uniruled for any $s \in S$.

Proof. By [8] the proposition is true if each point $s \in S$ admits a neighborhood $s \in U$ such that $f^{-1}(U)$ is Kähler. We shall prove the proposition by reducing to this result. Let $D=\{t \in \mathbb{C} ;|t|<1\}$ be the unit disc. Fix an arbitrary point $\xi \in D-\{0\}$. Then, since $S$ is connected, for any $s \in S$ we can find a finite number of morphisms $h_{i}: D \rightarrow S, i=1, \cdots, m$, such that $h_{1}(0)=0$, $h_{i}(\xi)=h_{i+1}(0), 1 \leqq i \leqq m-1$, and $h_{m}(\xi)=s$. Then it suffices to show inductively that $X_{h_{i}(\xi)}$ is uniruled for any $i$. Then the pull-back by $h_{i}$ reduces the proof to the case where $S=D$. In this case, however, $f$ satisfies the condition above by virtue of Proposition 5.

q.e.d.

Proof of Theorem. By Proposition $11 \mathfrak{A}$ is a union of connected compo- 
nents of $\mathfrak{M}$. In particular it is open in $\mathfrak{M}$. Since $\mathfrak{Y} \subseteq \mathfrak{M}-\mathfrak{R}$ by the definition of $\mathfrak{A}$ and $\mathfrak{R}$, by Proposition $10 \mathfrak{A}$ is separated. Finally from Proposition 4 it follows that $\mathfrak{X} \subseteq \mathfrak{B}$. Thus Theorem follows from Theorem 2.

(7.2) We shall give an immediate application of Theorem to local isomorphy problem of polarized families of compact Kähler manifolds.

Proposition 12. Let $\left(f_{i}: \mathscr{X}_{i} \rightarrow T, \widetilde{\omega}_{i}\right), i=1,2$, be polarized families of compact Kähler manifolds. Suppose that $X_{i, t}$ is not ruled for any $t \in T$, or $X_{i, t}$ is not uniruled for some $t \in T$ and $T$ is reduced and connected. Suppose further that there exists a dense subset $V \cong T$ such that $\left(X_{1 t}, \widetilde{\omega}_{1 t}\right) \cong\left(X_{2 t}, \widetilde{\omega}_{2 t}\right)$ for all $t \in V$. Then $\left(X_{1 t}, \widetilde{\omega}_{1 t}\right) \cong\left(X_{2 t}, \widetilde{\omega}_{2 t}\right)$ for all $t \in T$. Further if $T$ is locally irreducible, then the two families are locally isomorphic to each other.

Proof. By our assumption and by Proposition $11 \rho\left(f_{i}, \widetilde{\omega}_{i}\right)(|T|) \subseteq \mathfrak{B}-\Re$. Since $\mathfrak{B}-\mathfrak{R}$ is separated by Proposition 10 and since $\rho\left(f_{1}, \widetilde{\omega}_{1}\right)(t)=\rho\left(f_{2}, \widetilde{\omega}_{2}\right)(t)$ for all $t \in V, \rho\left(f_{1}, \widetilde{\omega}_{1}\right)=\rho\left(f_{2}, \widetilde{\omega}_{2}\right)$ on the whole $T$. This proves the first assertion. Next, suppose that $T$ is locally irreducible. Let $t \in T$ be an arbitrary point. Let $(f: \mathcal{X} \rightarrow S, \widetilde{\omega}),\left(X_{o}, \widetilde{\omega}_{o}\right)=\left(X_{1 t}, \widetilde{\omega}_{1 t}\right), o \in S$, be the local modular family of $\left(X_{1 t}, \widetilde{\omega}_{1 t}\right)$. We have the associated universal map $\tau_{i}: U \rightarrow S, \tau_{i}(t)=0, i$ $=1,2$ (with respect to some isomorphism $\left(X_{2 t}, \widetilde{\omega}_{2 t}\right) \cong\left(X_{1 t}, \widetilde{\omega}_{1 t}\right)$ for $\tau_{2}$ (cf. (5.3)), where $U$ is a small irreducible neighborhood of $t$. Let $\rho=\rho(f, \widetilde{\omega}): S \rightarrow \bar{S} \cong S / H$ be the canonical map, where $H:=H\left(X_{1 t}, \widetilde{\omega}_{1 t}\right)$. By the first assertion we have $\rho \tau_{1}=\rho \tau_{2}$. This implies that $\left(\tau_{1} \times \tau_{2}\right)(U) \subseteq R=\bigcup \mathcal{U}_{h \in H} \leqq S \times S$ in the notations of (6.2). Since $U$ is irreducible, $\left(\tau_{1} \times \tau_{2}\right)(U) \subseteq R^{h}$ for some $h$. Since $I^{h} \rightarrow R^{h}$ is smooth by Lemma $10, \tau_{1} \times \tau_{2}$ lifts to a morphism $\lambda: U \rightarrow I^{h}$. Hence the two families are isomorphic over $U$.

Remark 8. The final assertion is clearly false if $T$ is not locally irreducible (cf. [15, Cor. 7.3]). For a related result see [30].

We also prove the next result concerning a jumping phenomenon of complex structures, which is actually an application of Theorem 1.

Proposition 13. Let $D=\{t \in C ;|t|<1\}$ be the unit disc. Let $f: \mathfrak{X} \rightarrow D$ be a proper smooth morphism with connected fibers. Suppose that $X_{0}$ is Kähler and $X_{t}$ are isomorphic to each other for all $t \neq 0$. Then either $X_{0} \neq X_{t}, t \neq 0$, or $X_{0}$ is ruled.

Proof. Suppose that $X_{0} \nsucc X_{t}$. Then we have $\operatorname{dim} H^{0}\left(X_{0}, \Theta_{X_{0}}\right)>\operatorname{dim} H^{0}$ 
$\left(X_{t}, \Theta_{X_{t}}\right)$ for $t \neq 0$ (cf. [14, Prop. 5.6]). Then by Theorem 1 Aut $X_{0}$ is not a complex torus. Then by [6, Prop. 5.10] we see that $X_{0}$ is ruled.

q.e.d.

Note that the above proof gives more information than the proposition itself.

\section{§8. The Case of Complex Tori and K 3 Surfaces}

We are interested in the coarse moduli space for non-algebraic manifolds. Typical examples of such are complex tori and $\mathrm{K} 3$ surfaces. In these case the coarse moduli space can be obtained directly by considering the period maps. Let $\mathfrak{M}_{T}=\{(X, \omega) ; X$ is a complex torus $\}$ and $\mathfrak{M}_{K}=\{(X, \omega) ; X$ is a $\mathrm{K} 3$ surface\}. Then $\mathfrak{M}_{T}$ and $\mathfrak{M}_{K}$ are union of connected components of $\mathfrak{A}$ in Theorem. In this section we shall give a rather explicit description of the structure of the corresponding moduli spaces $\mathfrak{M}_{T}$ and $\mathfrak{M}_{K}$ by summarizing the known results on these spaces.

(8.1) Case of complex tori (cf. [15]). Fix once and for all a real vector space $W$ of even dimension $2 n$. Let $Q$ be a non-degenerate skew-symmetric bilinear form on $W$. Let $W_{\boldsymbol{C}}=W \otimes_{\boldsymbol{R}} C$. $Q$ extends naturally to $W_{\boldsymbol{C}}$. Let $\operatorname{Gr}\left(n, W_{\boldsymbol{C}}\right)$ be the Grassmann manifold of $n$-dimensional complex linear subspaces of $W_{C}$. We identify a point $S \in G r\left(n, W_{C}\right)$ with the corresponding subspace $S \subseteq W_{C}$. We define a submanifold $D_{Q}$ of $\operatorname{Gr}\left(n, W_{\boldsymbol{C}}\right)$ by

$$
D_{Q}=\left\{S \in G r\left(n, W_{C}\right) ; Q(S, S)=0, Q(S, \bar{S})>0\right\} .
$$

Here $Q(S, S)=0$ means that $Q\left(w_{1}, w_{2}\right)=0$ for any $w_{1}, w_{2} \in S$ and $Q(S, \bar{S})>0$ means that the Hermitian form $\sqrt{-1} Q\left(w_{1}, \bar{w}_{2}\right), w_{1}, w_{2} \in S$, is positive definite on $S$. Let $G_{Q}$ be the orthogonal group of $Q ; G_{Q}=\{g \in G L(W) ; Q(g w, g w)$ $=Q(w, w), w \in W\}$. Then $G_{Q}$ acts naturally on $D_{Q}$. Since $Q$ takes the form $Q=\left(\begin{array}{cc}0 & I_{n} \\ -I_{n} & 0\end{array}\right)$ with respect to a suitable base of $W$, the pair $\left(D_{Q}, G_{Q}\right)$ is isomorphic as a transformation space to the pair $\left(H_{n}, S p(n, \mathbb{R})\right)$, where $H_{n}$ is the Siegel upper half space of degree $n$ on which the real symplectic group $S p(n, \mathbb{R})$ acts in the usual manner. Fix now a lattice $A$ in $W$. Let

$$
\Gamma=\{g \in G L(W) ; g \Lambda=\Lambda\} \text { and } \Gamma_{Q}=\Gamma \cap G_{Q} .
$$

Then $\Gamma_{Q}$ is a discrete subgroup of $G_{Q}$ so that its action on $D_{Q}$ is properly discontinuous. Hence $M_{Q}:=D_{Q} / \Gamma_{Q}$ is naturally a normal analytic space.

Let $\mathfrak{Q}$ be the set of all nondegenerate skew-symmetric bilinear forms on 
$W$. $\mathfrak{Q}$ is a homogeneous space under the natural action of $G L(W)$; the stabilizer at $Q \in \mathfrak{Q}$ is just $G_{Q}$.

Proposition 14. For any $Q \in \mathfrak{Q}, M_{Q}$ is isomorphic to a connected component of $\mathfrak{M}_{T}$. Conversely, any connected component of $\mathfrak{M}_{T}$ is isomorphic to $M_{Q}$ for some $Q \in \mathfrak{Q}$ which is determined uniquely up to the action of $\Gamma$ on $Q$. Thus $M_{T}=\Perp_{Q} M_{Q}$, where the sum is taken over a complete set of representatives of $\mathfrak{\Omega} / \Gamma$.

We omit the proof, only indicating how one associates to each point of $M_{Q}$ a polarized complex torus. For each $S \in D_{Q}$ we have the direct sum decomposition $W_{C}=S \oplus \bar{S}$. Let $\Lambda_{S}$ be the projection of $\Lambda$ to $S$ which is a lattice in $S$. Then $T:=S / \Lambda_{S}$ is a complex torus with the natural isomorphism $W_{C} \equiv$ $\Lambda \otimes \boldsymbol{R} \cong H_{1}\left(T_{S}, \boldsymbol{R}\right)$, and then $Q$, as a skew-symmetric form on $W$, defines an element $\omega_{S} \in H^{2}\left(T_{S}, \boldsymbol{R}\right) \cong \wedge^{2} H^{1}\left(T_{S}, \mathbb{R}\right)$. Moreover the condition that $S \in D_{Q}$ implies that $\omega_{S}$ is a Kähler class. Then we associate to the point $\pi(S) \in M_{Q}$ the polarized complex torus $\left(T_{S}, \omega_{S}\right)$ whose isomorphism class is uniquely determined by $\pi(S)$, where $\pi: D_{Q} \rightarrow M_{Q}$ is the natural projection.

(8.2) Case of K3 surfaces. For general facts on K3 surfaces we refer the reader to [2] and [27]. Let $(L,\langle\rangle$,$) be an even unimodular Euclidian lattice$ of signature $(3,19)$, i.e., $L$ is a free abelian group of rank 22 and $\langle$,$\rangle is an$ even unimodular symmetric bilinear form on $L$ such that its extension to $L \otimes \boldsymbol{R}$ has signature $(3,19)$. Such an $(L,\langle\rangle$,$) is up to isomorphisms unique. Let$ $\boldsymbol{P}(L \otimes \boldsymbol{C})$ be the projective space of lines of $L \otimes \boldsymbol{C}$. Let $M=\{x \in \mathbb{P}(L \otimes \boldsymbol{C})$; $\langle x, x\rangle=0,\langle x, \bar{x}\rangle>0\}$, where the natural extension of $\langle$,$\rangle to L \otimes C$ is still denoted by $\langle$,$\rangle . M$ is a locally closed submanifold of $\boldsymbol{P}(L \otimes \boldsymbol{C})$ of codimension 1.

Let $V=\{\omega \in L \otimes R ;\langle\omega, \omega\rangle>0\} . \quad$ For any $a \in L \otimes \boldsymbol{R}$ we set $D_{a}=\{x \in M$; $\langle a, x\rangle=0\} . \quad D_{a}$ is an analytic submanifold of codimension 1 in $M$ if $a \neq 0$. Let $G_{0}=\{g \in G L(L \otimes \mathbb{R}) ;\langle g x, g x\rangle=\langle x, x\rangle, x \in L \otimes \mathbb{R}\}$. Let $G_{a}=\left\{g \in G_{0} ; g a\right.$ $=a\}$. Then $G_{a}$ acts naturally on $D_{a}$. Further if $a=\omega \in V$ we have an isomorphism $\left(D_{\omega}, G_{\omega}\right) \cong\left(D_{\mathrm{IV}}, O(2,19)\right)$ as transformation spaces, where $D_{\mathrm{IV}}$ is the bounded symmetric domain of type IV (cf. [14]). Let $\Gamma=G L(L) \subseteq$ $G L(L \otimes R), \Gamma_{0}=\Gamma \cap G_{0}$ and $\Gamma_{\omega}=G_{\omega} \cap \Gamma$. Then $\Gamma_{\omega}$ is a discrete subgroup of $G_{\omega}$ and hence acts properly discontinuously on $D_{\omega}$. Let $B_{\omega}=\{a \in L ;\langle a, a\rangle$ $=-2,\langle\omega, a\rangle=0\}$ and $U_{\omega}=D_{\omega}-\bigcup_{a \in B} D_{a}$.

Proposition 15. For any $\omega \in V, U_{\omega}$ is a $G_{\omega}$-invariant Zariski open subset 
of $D_{\omega}$ such that $U_{\omega} / \Gamma_{\omega}$ is naturally isomorphic to a connected component of $\mathfrak{M}_{K}$. Conversely, any connected component of $\mathfrak{M}_{K}$ is of this form for some $\omega \in V$ and $\omega$ is determined uniquely up to the natural action of $\Gamma_{0}$ on $V$; thus $\mathfrak{M}_{K}=\Perp_{\omega} U_{\omega} / \Gamma_{\omega}$, where the summation is over a complete set of representatives of $V / \Gamma_{0}$.

a. First we show that $U_{\omega}$ is Zariski open in $D_{\omega}$. Let $D_{\omega, a}=D_{\omega} \cap D_{a}$. Then it suffices to show that $\left\{D_{\omega, a}\right\}_{a \in B_{\omega}}$ is locally finite, i.e., for any $x \in D_{\omega}$ there exists a neighborhood $x \in W$ such that $\left\{a \in B_{\omega} ; D_{a} \cap W \neq \phi\right\}$ is a finite set. For any $x \in D_{\omega}$ let $x^{\prime}$ be any nonzero point on the line in $L \otimes C$ corresponding to $x$. Then $\operatorname{Re} x^{\prime}, \operatorname{Im} x^{\prime}$ and $\omega$ span a 3-dimensional subspace $F_{x}$ of $L \otimes R$ on which $\langle$,$\rangle is positive definite, where \mathrm{Re}, \mathrm{Im}$ denote the real and imaginary parts respectively (cf. [27]). Let $E_{x}=F_{x}^{\perp}$ be the orthogonal of $F_{x}$ with respect to $\langle$,$\rangle . Then \left\{E_{x}\right\}_{x \in D_{\omega}}$ is a $C^{\infty}$ family of subspaces of $L \otimes \mathbb{R}$ on which $\langle$, $\rangle$ is negative definite. Hence for any $x_{0} \in D_{\omega}$ and any relatively compact neighborhood $W$ of $x_{o}$ in $D_{\omega}, B_{\omega} \cap C_{W}$ is a finite set, where $C_{W}=\cup_{x \in W} \subseteq L \otimes R$. Thus the assertion follows since, for $x \in W$ and $a \in B_{\omega}, x \in D_{\omega, a}$ if and only if $a \in E_{x}$.

b. A marked $K 3$ surface is a pair $(X, \psi)$ consisting of a $\mathrm{K} 3$ surface and an isomorphism $\psi: H^{2}(X, Z) \rightarrow L$ of Euclidian lattices, where the bilinear form $\langle,\rangle_{X}$ on $H^{2}(X, \boldsymbol{Z})$ is given via the cup product. Define a functor $F:$ An $\rightarrow$ Sets by $F(S)=$ the set of isomorphism classes (in the obvious sense) of the pairs $(f: \mathfrak{X} \rightarrow S, \varphi)$, where $f$ is a proper smooth morphism of complex spaces with each fiber $X_{s}$ a K3 surface and $\varphi: R^{2} f_{*} Z \cong L \times S$ is a trivialization of the local system $R^{2} f_{*} \boldsymbol{Z}$. For any $(f, \varphi) \in F(S)$, let $\varphi_{\boldsymbol{R}}: R^{2} f_{*} \underset{R}{\longrightarrow}(L \otimes \boldsymbol{R}) \times S$ be the induced isomorphism. For any $\omega \in V$ let $\widetilde{\omega}=\varphi_{\boldsymbol{R}}^{-1}(\omega \times S) \in \Gamma\left(S, R^{2} f_{*} R\right)$. Let $\widetilde{\omega}_{s} \in H^{2}\left(X_{s}, \boldsymbol{R}\right)$ be induced by $\widetilde{\omega}$.

Define then the subfunctor $F_{\omega}$ of $F$ by $F_{\omega}(S)=\{(f, \varphi) \in F(S) ;(f, \widetilde{\omega})$ is a polarized family of Kähler K3 surfaces\}. A complex space which represents the functor $F_{\omega}$ will be called the fine moduli space of polarized marked K3 surfaces with polarization $\omega$.

Proposition 16. For any $\omega \in V$ the fine moduli space for polarized marked $K 3$ surfaces of polarization type $\omega$ exists and is naturally isomorphic to $U_{\omega}$ above.

Proof. By Burns-Rappoport [2] $F$ is represented by a 20-dimensional complex manifold $T$ which is not separated. Let $(f: \mathcal{X} \rightarrow T, \varphi)$ be the universal family. Then define the subspace $T_{\omega}$ of $T$ by $\eta(\widetilde{\omega})(t)=0$, where $\eta: R^{2} f_{*} \boldsymbol{R} \rightarrow$ $R^{2} f_{*} \mathcal{O}_{\mathcal{X}}$ is the natural homomorphism. Let $T_{\omega}^{\circ}=\left\{t \in T_{\omega} ; \widetilde{\omega}_{t}\right.$ is a Kähler class\}. Then $T_{\omega}^{\circ}$ is open in $T_{\omega}$ by Lemma 1 and it is immediate to see that $T_{\omega}^{\circ}$ 
represents $F_{\omega}$, where the universal family is the restriction of $(f, \varphi)$ to $T_{\omega}^{\circ}$. Let $p: T \rightarrow M$ be the period map associated to $(f, \varphi)$. Then for the second asser-

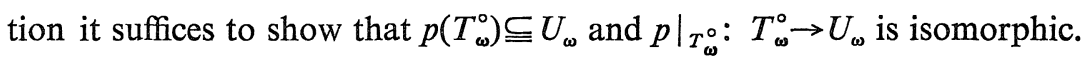

First, if $p(t) \in D_{a}$ for some $a \in B_{\omega}$, then $a_{t}:=\varphi_{t}^{-1}(a)$ is of type $(1,1)$ in $H^{2}\left(X_{t}, \mathbb{Z}\right)$ and then by Riemann-Roch either $a_{t}$ or $-a_{t}$ is represented by an effective curve. Then $\left\langle\widetilde{\omega}_{t}, a_{t}\right\rangle_{X_{t}} \neq 0$ because $\widetilde{\omega}_{t}$ is a Kähler class. This contradicts the fact that $a \in B_{\omega}$. Thus $p\left(T_{\omega}^{\circ}\right) \subseteq U_{\omega}$. Next, $p$ is locally an embedding by the local Torelli theorem (cf. [2]). Finally, the surjectivity can be seen as follows. For any $m \in U_{\omega}$, by Todorov [27] there exists a marked Kähler K3 surface $(X, \psi)$ with its period $m$. Since $m \in U_{\omega}$, by the proof of $[27,3.5]$, by changing the marking if necessary we may assume that $\psi^{-1}(\omega)$ belongs to the Kähler cone $V_{X}:=\left\{x \in H^{2}(X, \mathbb{R}) ;\langle x, x\rangle_{X}>0,\langle x, c\rangle_{X}>0\right.$ for any $c \in H^{2}$ $(X, \mathscr{Z})$ with $\langle c, c\rangle_{X}=-2$ which is represented by an effective curve on $\left.X\right\}$ of $X$. Then by Theorem 3 of [27] (cf. [20] for the proof) $\psi^{-1}(\omega)$ is a Kähler class, i.e., the point $t \in T$ corresponding to $(X, \psi)$ belongs to $T_{\omega}^{\circ}$ and we have $p(t)$ $=m$.

q.e.d.

c. From the above proposition and the global Torelli theorem the first and the second assertions of Proposition 15 follow. The last assertion follows from the next remark. Let $\mathfrak{M}_{K, \infty}$ be any connected component of $\mathfrak{M}_{K}$. Let $\Omega_{\omega}=\left\{\omega \in V ; \mathfrak{M}_{K, \alpha} \cong U_{\omega} / \Gamma_{\omega}\right\}$. Fix any $\left(X, \omega_{X}\right) \in \mathfrak{M}_{K, \alpha}$. Then $\omega \in \Omega_{\alpha}$ if and only if there exists a marking $\psi: H^{2}(X, \mathbb{Z}) \rightarrow L$ such that $\psi_{R}\left(\omega_{X}\right)=\omega$.

Remark 9. In Propositions 14 and $15 \Gamma$ is countable so that for 'general' $Q \in \mathfrak{Q}$ (resp. $\omega \in V$ ), the corresponding $\Gamma_{Q}$ (resp. $\Gamma_{\omega}$ ) reduces to the identity and hence $M_{Q} \cong H_{n}$ (resp. $M_{\omega} \cong D_{\text {IV }}$ ). In particular there is no analytic compactification of $M_{Q}$ or $M_{\omega}$ in contrast to the algebraic case where the Baily-Borel compactification is available.

\section{References}

[1] Banica, C. and Stanasila, O., Algebraic methods in the global theory of complex spaces, John Wiley \& Sons and Editura Academiei, 1976.

[2] Burns, D. and Rappoport, M., On the Torelli problem for kählerian K3 surfaces, Ann. Sci. Ec. Norm. Sup., 8 (1975), 235-274.

[ 3 ] Deligne, P., Théorème de Lefschetz et critère de dégénérescence de suites spectrals, Publ. Math. I.H.E.S., 35 (1968), 107-126.

[4] Douady, A., Le problème de modules pour les sous-espaces analytiques complexes d'un espace analytique donné, Ann. Inst. Fourier, Grenoble, 16 (1966), 1-95.

[5] Fujiki, A., Closedness of the Douady spaces of compact Kähler spaces, Publ. RIMS, Kyoto Univ., 14 (1978), 1-52. 
[ 6 ] - On automorphism groups of compact Kähler manifolds, Inventiones math., 44 (1978), 225-258.

[ 7 ] — Moduli space for polarized compact Kähler manifolds, Sûriken-kôkyûroku, 387 (1980), 1-17, (in Japanese).

[ 8 ] —, Deformations of uniruled manifolds, Publ. RIMS, Kyoto Univ., 17 (1981), 687-702.

[9] — A theorem on bimeromorphic maps of Kähler manifolds and its applications, Publ. RIMS, Kyoto Univ., 17 (1981), 735-754.

[10] $\longrightarrow$ On a holomorphic fiber bundle with meromorphic structure, Publ. RIMS, Kyoto Univ., 19 (1983), 117-134.

[11] $\longrightarrow$, On the Douady space of a compact complex space in the category $\mathcal{C}, \mathrm{II}$, Publ. RIMS, Kyoto Univ., 20 (1984), 461-489.

[12] — C Coarse moduli space for polarized algebraic manifolds, to appear.

[13] - On the formal Poincare and Dolbeault lemmas on a complex space, Sûriken-kĉkyûroku, 372 (1979), 1-16, (in Japanese).

[14] Griffiths, P.A., The extension problem for compact submanifolds I, in Proc. Conf. on Complex Analysis, Minneapolis, 1974, 113-142.

[15] — Periods of integrals on algebraic manifolds I, Amer. J. Math., 90 (1968), $568-626$.

[16] Grothendieck, A., Technique de construction en géométrie analytique, Séminaire $H$. Cartan, 13 année, 1960/61.

[17] Hirshowitz, A., Sur les plongements du type deformation, Comment. Math. Helvetici, 54 (1979), 126-132.

[18] Kodaira, K. and Morrow, J., Complex manifolds, Holt, Reinhart and Winston, 1971.

[19] Kuranishi, M., Deformations of compact complex manifolds, Les presse de l'université de Montreal, 1971.

[20] Looijenga, E., A Torelli theorem for Kähler-Einstein K3 surfaces, Lecture Notes in Math., 894, 107-112, Springer, 1980.

[21] Matsusaka, T., Algebraic deformations of polarized varieties, Nagoya Math. J., 31 (1968), 185-245.

[22] Matsusaka, T. and Mumford, D., Two fundamental theorems on deformations of polarized varieties, Amer. J. Math., 86 (1964), 668-684.

[23] Mumford, D., Geometric invariant theory, Ergeb. der Math., 34, Springer, 1965.

[24] Narasimhan, M.S. and Shimha, R.R., Manifolds with ample canonical class, Inventiones math., 5 (1968), 120-128.

[25] Palamodov, V.P., Moduli in versal deformations of complex spaces, In: Variétés analytiques compactes, Lecture Notes in Math., 683, 74-115, Springer, 1978.

[26] Schuster, H.W., Zur Theorie der Deformationen kompakter komplexer Räume, Inventiones math., 9 (1970), 284-294.

[27] Todorov, A.N., Applications of the Kähler-Einstein-Calabi-Yau metric to moduli of K3 surfaces, Inventiones math., 61 (1980), 251-265.

[28] Tjurina, G.N., The space of moduli of a complex surface with $q=0$ and $K=0$, in Savarevic, Algebraic surfaces, Steklov Inst. of Math., 75, English translation, Providence, Amer. Math. Soc., 1967, 193-225.

[29] Wavrik, J.J., Obstructions to the existence of a space of moduli, in Global Analysis, Papers in Honor of K. Kodaira, 403-414, 1969.

[30] Wehler, J., Isomorphie von Familien kompakter komplexer Mannigfaltigkeiten, Math. Ann., 231 (1977), 77-90.

[31] Bingener, J., Über formale komplexe Räume, Manuscripta math., 24 (1978), 253293. 
